\title{
Ramanujan identities and Euler products for a type of Dirichlet series
}

\author{
by
}

\section{Zhi-Hong Sun (Huaian) and Kenneth S. Williams (Ottawa)}

1. Introduction. In his lost notebook [R2] Ramanujan stated that the Dirichlet series $\sum_{n=1}^{\infty} a(n) n^{-s}(\operatorname{Re}(s)>1)$, where the Dirichlet coefficients $a(n)(n=1,2, \ldots)$ are given by

$$
q \prod_{n=1}^{\infty}\left(1-q^{2 n}\right)\left(1-q^{22 n}\right)=\sum_{n=1}^{\infty} a(n) q^{n} \quad(|q|<1),
$$

has an Euler product and gave an explicit formulation for the Euler product. In this paper we develop the theory of binary quadratic forms in order to determine the Euler product for $\sum_{n=1}^{\infty} a(n) n^{-s}$, and other similarly defined Dirichlet series, in a completely elementary and natural manner.

Let $\mathbb{N}, \mathbb{Z}, \mathbb{R}$ and $\mathbb{C}$ be the sets of natural numbers, integers, real numbers and complex numbers respectively. A nonsquare integer $d$ with $d \equiv 0,1$ $(\bmod 4)$ is called a discriminant. The conductor of the discriminant $d$ is the largest positive integer $f=f(d)$ such that $d / f^{2} \equiv 0,1(\bmod 4)$. As usual we set $w(d)=1,2,4,6$ according as $d>0, d<-4, d=-4$ or $d=-3$.

For integers $a, b$ and $c$, we use $(a, b, c)$ to denote the integral, binary quadratic form $a x^{2}+b x y+c y^{2}$. The form $(a, b, c)$ is said to be primitive if $\operatorname{gcd}(a, b, c)=1$. The discriminant of the form $(a, b, c)$ is the integer $d=$ $b^{2}-4 a c$. If $d<0$, we only consider positive definite forms, that is, forms $(a, b, c)$ with $a>0$ and $c>0$. Two forms $(a, b, c)$ and $\left(a^{\prime}, b^{\prime}, c^{\prime}\right)$ are equivalent $\left((a, b, c) \sim\left(a^{\prime}, b^{\prime}, c^{\prime}\right)\right)$ if there exist integers $\alpha, \beta, \gamma$ and $\delta$ with $\alpha \delta-\beta \gamma=1$ such that the substitution $x=\alpha X+\beta Y, y=\gamma X+\delta Y$ transforms $(a, b, c)$ to $\left(a^{\prime}, b^{\prime}, c^{\prime}\right)$. It is known that $(a, b, c) \sim(c,-b, a)$ and for $k \in \mathbb{Z}$ that $(a, b, c) \sim$ $\left(a, 2 a k+b, a k^{2}+b k+c\right)$. We denote the equivalence class of $(a, b, c)$ by $[a, b, c]$. The equivalence classes of primitive, integral, binary quadratic forms

2000 Mathematics Subject Classification: 11E16, 11F66, 11E25, $11 \mathrm{~B} 65$.

The first author was supported by Natural Sciences Foundation of Jiangsu Educational Office (02KJB110007), and the second author was supported by Natural Sciences and Engineering Research Council of Canada grant A-7233. 
of discriminant $d$ form a finite abelian group under Gaussian composition, called the form class group. We denote this group by $H(d)$ and its order by $h(d)$. The identity of $H(d)$ is the so-called principal class $[1,0,-d / 4]$ or $[1,1,(1-d) / 4]$ according as $d \equiv 0(\bmod 4)$ or $d \equiv 1(\bmod 4)$, and the inverse of the class $K=[a, b, c]$ is the class $K^{-1}=[a,-b, c]$.

Let $(a, b, c)$ be an integral, binary quadratic form of discriminant $d$. The positive integer $n$ is said to be represented by $(a, b, c)$ if there exist integers $x$ and $y$ with $n=a x^{2}+b x y+c y^{2}$, and the pair $\{x, y\}$ is called a representation. If $d<0$, every representation $\{x, y\}$ is called primary. If $d>0$, the representation $\{x, y\}$ is called primary if it satisfies

$$
2 a x+(b-\sqrt{d}) y>0 \text { and } 1 \leq\left|\frac{2 a x+(b+\sqrt{d}) y}{2 a x+(b-\sqrt{d}) y}\right|<\varepsilon(d)^{2},
$$

where $\varepsilon(d)=\left(x_{1}+y_{1} \sqrt{d}\right) / 2$ and $\left(x_{1}, y_{1}\right)$ is the solution in positive integers to the equation $X^{2}-d Y^{2}=4$ for which $\left(x_{1}+y_{1} \sqrt{d}\right) / 2$ (or equivalently $y_{1}$ ) is least (see [Di], $[\mathrm{H}]$ ). For $a, b, c \in \mathbb{Z}$ and $n \in \mathbb{N}$ we define

$$
R(a, b, c ; n)=\mid\left\{\{x, y\} \mid n=a x^{2}+b x y+c y^{2},\{x, y\} \text { is primary }\right\} \mid .
$$

If $(a, b, c) \sim\left(a^{\prime}, b^{\prime}, c^{\prime}\right)$, by [SW, Remark 3.1] we have

$$
R(a, b, c ; n)=R(a,-b, c ; n)=R\left(a^{\prime}, b^{\prime}, c^{\prime} ; n\right) .
$$

From this we define $R([a, b, c], n)=R(a, b, c ; n)$ as in $[\mathrm{SW}]$.

Let $d$ be a discriminant. Suppose $H(d)=\left\{A_{1}^{k_{1}} \cdots A_{r}^{k_{r}} \mid 0 \leq k_{1}<\right.$ $\left.h_{1}, \ldots, 0 \leq k_{r}<h_{r}\right\}$ with $h_{1} \cdots h_{r}=h(d)$. For $n \in \mathbb{N}$ and $M=A_{1}^{m_{1}} \cdots A_{r}^{m_{r}}$ $\in H(d)$, following [SW, Definition 7.1] we define

$$
\begin{aligned}
& F(M, n) \\
& =\frac{1}{w(d)} \sum_{\substack{0 \leq k_{1}<h_{1} \\
\cdots, k_{r}<h_{r} \\
0 \leq k_{r}}} \cos 2 \pi\left(\frac{k_{1} m_{1}}{h_{1}}+\cdots+\frac{k_{r} m_{r}}{h_{r}}\right) \cdot R\left(A_{1}^{k_{1}} \cdots A_{r}^{k_{r}}, n\right) .
\end{aligned}
$$

In particular, if $h(d)=2,3,4$ and $H(d)$ is cyclic with principal class $I$ and generator $A$, then (see [SW, Theorem 7.4])

$$
F(A, n)= \begin{cases}\frac{1}{w(d)}(R(I, n)-R(A, n)) & \text { if } h(d)=2,3, \\ \frac{1}{w(d)}\left(R(I, n)-R\left(A^{2}, n\right)\right) & \text { if } h(d)=4 .\end{cases}
$$

Let $s \in \mathbb{C}$ with $\operatorname{Re}(s)>1$. In this paper we introduce

$$
L(M, s)=\sum_{n=1}^{\infty} \frac{F(M, n)}{n^{s}} \quad \text { for } M \in H(d) .
$$


From [SW, Theorem 7.2] we know that $F(M, n)$ is a multiplicative function of $n \in \mathbb{N}$. Thus, if $\operatorname{Re}(s)>1$, then

$$
L(M, s)=\prod_{p}\left(1+\sum_{t=1}^{\infty} F\left(M, p^{t}\right) p^{-s t}\right),
$$

where $p$ runs over all primes.

From (1.5) we see that $L(M, s)$ has an Euler product. The main purpose of this paper is to give the Euler product for $L(M, s)$. When $H(d)$ is cyclic, in Section 5 we completely determine the Euler product for $L(M, s)$ $(M \in H(d), \operatorname{Re}(s)>1)$, see Theorem 5.3. As consequences, in Sections 6-8 we give explicit Euler products for $L(M, s)$ in the cases $h(d)=2,3$ and $H(d)$ is cyclic of order 4 .

For $|q|<1$ let $\psi(q)$ and $\phi(q)$ be the theta functions defined by

$$
\begin{aligned}
& \psi(q)=\sum_{n=0}^{\infty} q^{n(n+1) / 2}, \\
& \phi(q)=\prod_{m=1}^{\infty}\left(1-q^{m}\right)=\sum_{n=-\infty}^{\infty}(-1)^{n} q^{\left(3 n^{2}-n\right) / 2} .
\end{aligned}
$$

Ramanujan (see for example [B]) established many identities involving $\psi(q)$ and $\phi(q)$. In Section 4 of this paper we prove some of these identities from our point of view.

For $k=1, \ldots, 12$ let

$$
q \phi\left(q^{k}\right) \phi\left(q^{24-k}\right)=\sum_{n=1}^{\infty} \phi_{k}(n) q^{n} \quad(|q|<1) .
$$

Ramanujan ([R1], [R2]) conjectured that the Dirichlet series $\sum_{n=1}^{\infty} \frac{\phi_{k}(n)}{n^{s}}$ $(k=1,2,3,4,6,8,12)$ have Euler products and gave the explicit Euler products in the cases $k=1,2,3$. Unfortunately his formulae for $k=2,3$ are wrong. In [Ra] Rangachari outlined the proofs of the formulae for $k=1,2,3$ using class field theory and modular forms. But Rangachari's formulae for $k=2,3$ are also wrong and his proofs are neither clear nor elementary. So it remains to correct the results and to give elementary proofs of them. For instance, the corrected form of Ramanujan's conjecture in his lost notebook $([\mathrm{R} 2])$ is

$$
\begin{aligned}
& \sum_{n=1}^{\infty} \frac{\phi_{2}(n)}{n^{s}}=\frac{1}{1-11^{-s}} \prod_{\substack{p \equiv 2,6,7,8,10(\bmod 11) \\
p \neq 2}} \frac{1}{1-p^{-2 s}} \\
& \times \prod_{p=3 x^{2}+2 x y+4 y^{2}} \frac{1}{1+p^{-s}+p^{-2 s}} \prod_{p=x^{2}+11 y^{2} \neq 11} \frac{1}{\left(1-p^{-s}\right)^{2}},
\end{aligned}
$$


where $p$ runs over all primes. We note that this formula also corrects the incorrect formula in [Ra].

Let $\delta_{k}=\left(1-(-1)^{k}\right) / 2$ and $n \in \mathbb{N}$. In Section 2 we show that for $k=1, \ldots, 12$,

$$
\phi_{k}(n)=\frac{1}{2}\left(R\left(1, \delta_{k},\left(24 k-k^{2}+\delta_{k}\right) / 4 ; n\right)-R(4,4-k, k+1 ; n)\right) .
$$

Moreover, we obtain explicit formulas for $\phi_{k}(n)$ in the cases $k=1,2,3,4,6$, 8,12 (see Theorems 4.4 and 4.5). From the above it follows that for $k=$ $1,2,3,4,6,8,12$,

$$
\sum_{n=1}^{\infty} \frac{\phi_{k}(n)}{n^{s}}=L\left(A_{k}, s\right) \quad \text { for } \operatorname{Re}(s)>1,
$$

where $A_{k}=[2,1,3],[3,2,4],[2,1,8],[3,2,7],[4,2,7],[3,2,11],[5,4,8]$ according as $k=1,2,3,4,6,8,12$. Thus using the results for Dirichlet series $L(M, n)$ we obtain the Euler products for $\sum_{n=1}^{\infty} \phi_{k}(n) n^{-s}$ in the cases $k=1,2,3,4,6,8,12$. In this way we prove all of Ramanujan conjectures for $\phi_{k}(n)$, and our proofs are natural and elementary. It seems that Ramanujan's conjecture for $\phi_{12}(n)$ was first proved by Mordell ([M]).

We should mention that the Euler products for $\sum_{n=1}^{\infty} \phi_{k}(n) n^{-s}$ in the cases $k=1,2,3,4,6,8,12$ are connected with modular forms (see for example $[\mathrm{Ra}])$.

In addition to the above notation, we also use throughout this paper the following notation: $\operatorname{ord}_{p} n$ denotes the nonnegative integer $\alpha$ such that $p^{\alpha} \mid n$ but $p^{\alpha+1} \nmid n, p^{\alpha} \| n$ means $p^{\alpha} \mid n$ but $p^{\alpha+1} \nmid n,\left(\frac{a}{m}\right)$ is the Kronecker symbol, $(a, b)$ is the greatest common divisor of the integers $a$ and $b$ (not both zero), $I$ denotes the principal class in $H(d)$, and $R(K)$ denotes the set of integers represented by forms in the class $K$.

Throughout this paper $p$ denotes a prime and products (sums) over $p$ run through all primes $p$ satisfying any restrictions given under the product (summation) symbol. For example the condition $p=x^{2}+11 y^{2}$ under a product restricts the product to those primes $p$ which are of the form $x^{2}+$ $11 y^{2}$ for some integers $x$ and $y$.

2. Generating functions for $\frac{1}{2}(R(I, n)-R(K, n))$ when $I, K \in$ $H(d)$. For $q \in \mathbb{R}, m \in \mathbb{N}$ and $r \in \mathbb{Z}$ let

$$
f(r, m ; q)=\sum_{n=-\infty}^{\infty}(-1)^{n} q^{\left(m n^{2}-r n\right) / 2} \quad(|q|<1) .
$$

From Jacobi's identity (cf. [HW, Theorem 352, p. $282\left(\right.$ with $x=q^{m / 2}, z=$ $\left.\left.-q^{-r / 2}\right)\right]$ ) we know that 
(2.2) $\quad f(r, m ; q)$

$$
=\prod_{n=0}^{\infty}\left\{\left(1-q^{m n+(m-r) / 2}\right)\left(1-q^{m n+m}\right)\left(1-q^{m n+(m+r) / 2}\right)\right\} \quad(|q|<1) .
$$

Definition 2.1. For $r \in \mathbb{Z}, m \in \mathbb{N}$ and $k \in\{1, \ldots, 4 m\}$ with $2 \mid k(m-r)$ we define

$$
q^{r^{2}} f\left(r, m ; q^{k}\right) f\left(r, m ; q^{8 m-k}\right)=\sum_{n=0}^{\infty} f_{k}(r, m ; n) q^{n} .
$$

Proposition 2.1. Let $r \in \mathbb{Z}$ and let $k, m, n \in \mathbb{N}$ with $k \leq 4 m$ and $2 \mid k(m-r)$. Then

$$
\begin{aligned}
& f_{k}(r, m ; n)
\end{aligned}
$$

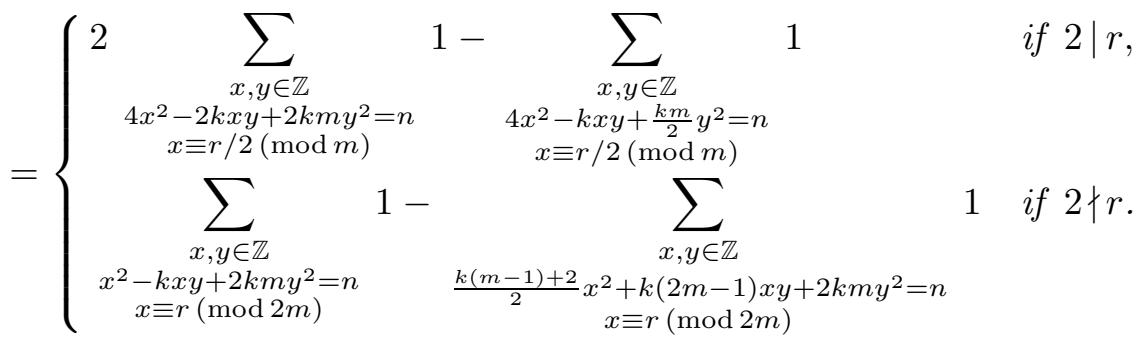

Proof. From (2.1) and Definition 2.1 we have

$$
f_{k}(r, m ; n)=\sum_{\substack{x, y \in \mathbb{Z} \\ \frac{k\left(m x^{2}-r x\right)}{2}+\frac{(8 m-k)\left(m y^{2}-r y\right)}{2}+r^{2}=n}}(-1)^{x+y} .
$$

It is clear that

$$
\begin{aligned}
16\left(\frac{k\left(m x^{2}-r x\right)}{2}+\frac{(8 m-k)\left(m y^{2}-r y\right)}{2}+r^{2}\right) & \\
& =(k x+(8 m-k) y-4 r)^{2}+k(8 m-k)(x-y)^{2} .
\end{aligned}
$$

Thus

$$
\begin{gathered}
f_{k}(r, m ; n)=\sum_{\substack{x, y \in \mathbb{Z} \\
(k x+(8 m-k) y-4 r)^{2}+k(8 m-k)(x-y)^{2}=16 n}}(-1)^{x-y} \\
=\sum_{\substack{y, z \in \mathbb{Z} \\
(8 m y+k z-4 r)^{2}+k(8 m-k) z^{2}=16 n}}^{(-1)^{z}} \\
=\sum_{\substack{x, z \in \mathbb{Z} \\
x^{2}+k(8 m-k) z^{2}=16 n \\
x \equiv k z-4 r(\bmod 8 m)}}(-1)^{z}=\sum_{\substack{y, z \in \mathbb{Z} \\
y^{2}+2 k y z+8 k m z^{2}=16 n \\
y \equiv-4 r(\bmod 8 m)}}^{z}
\end{gathered}
$$




$$
=\sum_{\substack{x, z \in \mathbb{Z} \\ 16 x^{2}-8 k x z+8 k m z^{2}=16 n \\-4 x \equiv-4 r(\bmod 8 m)}}(-1)^{z}=\sum_{\substack{x, y \in \mathbb{Z} \\ 2 x^{2}-k x y+k m y^{2}=2 n \\ x \equiv r(\bmod 2 m)}}(-1)^{y} .
$$

If $r$ is even, then $2 \mid k m$. By the above we must have

$$
\begin{aligned}
& f_{k}(r, m ; n)=\sum_{\substack{x, y \in \mathbb{Z} \\
2(2 x)^{2}-k(2 x) y+k m y^{2}=2 n \\
2 x \equiv r(\bmod 2 m)}}(-1)^{y}=\sum_{\substack{x, y \in \mathbb{Z} \\
4 x^{2}-k x y+\frac{k m}{2} \\
x \equiv r / 2(\bmod m)}}(-1)^{y} \\
& =2 \sum_{\begin{array}{c}
x, y \in \mathbb{Z} \\
4 x^{2}-2 k x y+2 k m y^{2}=n \\
x \equiv r / 2(\bmod m)
\end{array}} 1-\sum_{\begin{array}{c}
x, y \in \mathbb{Z} \\
4 x^{2}-k x y+\frac{k m}{2} y^{2}=n \\
x \equiv r / 2(\bmod m)
\end{array}} 1 .
\end{aligned}
$$

If $r$ is odd, then $2 \mid k(m-1)$. From the above we obtain

$$
\begin{aligned}
& f_{k}(r, m ; n) \\
& =\sum_{\begin{array}{c}
x, y \in \mathbb{Z} \\
2 x^{2}-k x y+k m y^{2}=2 n \\
x \equiv r(\bmod 2 m), 2 \mid y
\end{array}} 1-\sum_{\begin{array}{c}
x, y \in \mathbb{Z} \\
2 x^{2}-k x y+k m y^{2}=2 n \\
x \equiv r(\bmod 2 m), 2 \nmid y
\end{array}} 1 \\
& =\sum_{\substack{x, y \in \mathbb{Z} \\
2 x^{2}-2 k x y+4 k m y^{2}=2 n \\
x \equiv r(\bmod 2 m)}} 1-\sum_{\substack{x, y \in \mathbb{Z} \\
(k m-k+2)}} \\
& =\sum_{\substack{x, y \in \mathbb{Z} \\
x^{2}-k x y+2 k m y^{2}=n \\
x \equiv r(\bmod 2 m)}} 1-\sum_{\substack{x, y \in \mathbb{Z} \\
(k m-k+2) \\
x^{2}+k(2 m-1) x(2 y)+k m(2 y)^{2}=2 n \\
x \equiv r(\bmod 2 m)}} \\
& =\sum_{\begin{array}{c}
x, y \in \mathbb{Z} \\
x^{2}-k x y+2 k m y^{2}=n \\
x \equiv r(\bmod 2 m)
\end{array}} 1-\sum_{\substack{x, y \in \mathbb{Z} \\
x^{2}+k(2 m-1) x y+2 k m y^{2}=n \\
x \equiv r(\bmod 2 m)}}
\end{aligned}
$$

This finishes the proof.

Definition 2.2. Let $\psi(q)=\sum_{n=0}^{\infty} q^{n(n+1) / 2}$. For $k \in\{1,2,3,4\}$ we define $\psi_{k}(n)$ by

$$
q \psi\left(-q^{k}\right) \psi\left(-q^{8-k}\right)=\sum_{n=1}^{\infty} \psi_{k}(n) q^{n} \quad(|q|<1) .
$$

TheOREM 2.1. For $k \in\{1,2,3,4\}$ and $n \in \mathbb{N}$ we have

$$
\psi_{k}(n)=\frac{1}{2}(R(1,0, k(8-k) ; n)-R(4,4-2 k, k+1 ; n)) .
$$


Proof. Since

$$
\begin{aligned}
\sum_{n=0}^{\infty} q^{n(n+1) / 2} & =1+\sum_{n=1}^{\infty} q^{(2 n-1)(2 n) / 2}+\sum_{n=1}^{\infty} q^{2 n(2 n+1) / 2} \\
& =\sum_{n=-\infty}^{\infty} q^{2 n^{2}-n} \quad(|q|<1)
\end{aligned}
$$

we see that

$$
\begin{aligned}
\sum_{n=1}^{\infty} \psi_{k}(n) q^{n} & =q \psi\left(-q^{k}\right) \psi\left(-q^{8-k}\right) \\
& =q\left(\sum_{n=-\infty}^{\infty}\left(-q^{k}\right)^{2 n^{2}-n}\right)\left(\sum_{n=-\infty}^{\infty}\left(-q^{8-k}\right)^{2 n^{2}-n}\right) \\
& =q\left(\sum_{n=-\infty}^{\infty}(-1)^{n} q^{k\left(2 n^{2}-n\right)}\right)\left(\sum_{n=-\infty}^{\infty}(-1)^{n} q^{(8-k)\left(2 n^{2}-n\right)}\right) \\
& =q f\left(1,2 ; q^{2 k}\right) f\left(1,2 ; q^{16-2 k}\right)=\sum_{n=0}^{\infty} f_{2 k}(1,2 ; n) q^{n} \quad(|q|<1) .
\end{aligned}
$$

Thus $\psi_{k}(n)=f_{2 k}(1,2 ; n)$. Applying Proposition 2.1 we obtain

$$
\begin{aligned}
& \psi_{k}(n)=\sum_{\begin{array}{c}
x, y \in \mathbb{Z} \\
x^{2}-2 k x y+8 k y^{2}=n \\
x \equiv 1(\bmod 4)
\end{array}} 1-\sum_{\begin{array}{c}
x, y \in \mathbb{Z} \\
(k+1) x^{2}+6 k x y+8 k y^{2}=n \\
x \equiv 1(\bmod 4)
\end{array}} 1 \\
& =\frac{1}{2}\left(\sum_{\substack{x, y \in \mathbb{Z} \\
x^{2}-2 k x y+8 k y^{2}=n}} 1-\sum_{\substack{x, y \in \mathbb{Z} \\
x^{2}-2 k x y+8 k y^{2}=n \\
2 \mid x}} 1\right) \\
& -\frac{1}{2}\left(\sum_{\substack{x, y \in \mathbb{Z} \\
(k+1) x^{2}+6 k x y+8 k y^{2}=n}} 1-\sum_{\substack{x, y \in \mathbb{Z} \\
(k+1) x^{2}+6 k x y+8 k y^{2}=n \\
2 \mid x}} 1\right) \\
& =\frac{1}{2}(R(1,-2 k, 8 k ; n)-R(4,-4 k, 8 k ; n) \\
& -R(k+1,6 k, 8 k ; n)+R(4 k+4,12 k, 8 k ; n)) \text {. }
\end{aligned}
$$

Note that $(4 k+4,12 k, 8 k) \sim(8 k,-12 k, 4 k+4) \sim(8 k, 4 k, 4) \sim(4,-4 k, 8 k)$, $(1,-2 k, 8 k) \sim(1,0, k(8-k))$ and $(k+1,6 k, 8 k) \sim(k+1,2 k-4,4) \sim$ $(4,4-2 k, k+1)$. We then obtain the desired result. 
Definition 2.3. Let $\phi(q)=\sum_{n=-\infty}^{\infty}(-1)^{n} q^{\left(3 n^{2}-n\right) / 2}(|q|<1)$. For $k \in\{1, \ldots, 12\}$ define $\phi_{k}(n)$ by

$$
\sum_{n=1}^{\infty} \phi_{k}(n) q^{n}=q \phi\left(q^{k}\right) \phi\left(q^{24-k}\right) \quad(|q|<1) .
$$

As $\phi(q)=f(1,3 ; q)$ it is clear that $\phi_{k}(n)=f_{k}(1,3 ; n)$.

Theorem 2.2. Let $k \in\{1, \ldots, 12\}$ and $\delta_{k}=\left(1-(-1)^{k}\right) / 2$. For $n \in \mathbb{N}$ we have

$$
\begin{aligned}
& \phi_{k}(n)=\frac{1}{2}\left(R\left(1, \delta_{k},\left(24 k-k^{2}+\delta_{k}\right) / 4 ; n\right)-R(4,4-k, k+1 ; n)\right) \\
& = \begin{cases}\frac{1}{2}(R(1,1,6 ; n)-R(2,1,3 ; n)) & \text { if } k=1, \\
\frac{1}{2}\left(R\left(1, \delta_{k},\left(24 k-k^{2}+\delta_{k}\right) / 4 ; n\right)-R(k+1,4-k, 4 ; n)\right) & \text { if } 2 \leq k \leq 3, \\
\frac{1}{2}\left(R\left(1, \delta_{k},\left(24 k-k^{2}+\delta_{k}\right) / 4 ; n\right)-R(4, k-4, k+1 ; n)\right) & \text { if } 4 \leq k \leq 8, \\
\frac{1}{2}\left(R\left(1, \delta_{k},\left(24 k-k^{2}+\delta_{k}\right) / 4 ; n\right)-R(4,12-k, 9 ; n)\right) & \text { if } 9 \leq k \leq 12 .\end{cases}
\end{aligned}
$$

Proof. As $\phi_{k}(n)=f_{k}(1,3 ; n)$, by Proposition 2.1 we have

$$
\phi_{k}(n)=\sum_{\substack{x, y \in \mathbb{Z} \\
x^{2}-k x y+6 k y^{2}=n \\
x \equiv 1(\bmod 6)}} 1-\sum_{\begin{array}{c}
x, y \in \mathbb{Z} \\
(k+1) x^{2}+5 k x y+6 k y^{2}=n \\
x \equiv 1(\bmod 6)
\end{array}} 1 .
$$

Since

$$
\begin{aligned}
& \sum_{\substack{x, y \in \mathbb{Z} \\
a x^{2}+b x y+c y^{2}=n \\
x \equiv 1(\bmod 6)}} 1 \\
= & \sum_{\substack{x, y \in \mathbb{Z} \\
a x^{2}+b x y+c y^{2}=n \\
2 \nmid x, x \equiv 1(\bmod 3)}} 1=\sum_{\substack{x, y \in \mathbb{Z} \\
a x^{2}+b x y+c y^{2}=n \\
x \equiv 1(\bmod 3)}} 1-\sum_{\substack{x, y \in \mathbb{Z} \\
4 a x^{2}+2 b x y+c y^{2}=n \\
x \equiv 1(\bmod 3)}} 1 \\
= & \frac{1}{2}\left(\sum_{\substack{x, y \in \mathbb{Z} \\
a x^{2}+b x y+c y^{2}=n}} 1-\sum_{\substack{x, y \in \mathbb{Z} \\
9 a x^{2}+3 b x y+c y^{2}=n}} 1\right) \\
& -\frac{1}{2}\left(\sum_{\substack{x, y \in \mathbb{Z} \\
4 a x^{2}+2 b x y+c y^{2}=n}} \sum_{\substack{x, y \in \mathbb{Z} \\
36 a x^{2}+6 b x y+c y^{2}=n}} 1\right) \\
= & \frac{1}{2}(R(a, b, c ; n)-R(9 a, 3 b, c ; n)-R(4 a, 2 b, c ; n)+R(36 a, 6 b, c ; n)),
\end{aligned}
$$

we see that 


$$
\begin{aligned}
2 \phi_{k}(n)= & (R(1,-k, 6 k ; n)-R(9,-3 k, 6 k ; n)-R(4,-2 k, 6 k ; n) \\
& +R(36,-6 k, 6 k ; n))-(R(k+1,5 k, 6 k ; n)-R(9 k+9,15 k, 6 k ; n) \\
& -R(4 k+4,10 k, 6 k ; n)+R(36 k+36,30 k, 6 k ; n)) .
\end{aligned}
$$

Note that $(9 k+9,15 k, 6 k) \sim(6 k,-15 k, 9 k+9) \sim(6 k,-3 k, 9) \sim(9,3 k, 6 k)$, $(36 k+36,30 k, 6 k) \sim(6 k,-30 k, 36 k+36) \sim(6 k, 6 k, 36) \sim(36,-6 k, 6 k)$ and $(4 k+4,10 k, 6 k) \sim(4 k+4,2 k-8,4) \sim(4,8-2 k, 4 k+4) \sim(4,-2 k, 6 k)$. We find $R(9 k+9,15 k, 6 k ; n)=R(9,-3 k, 6 k ; n), R(36 k+36,30 k, 6 k ; n)=$ $R(36,-6 k, 6 k ; n)$ and $R(4 k+4,10 k, 6 k ; n)=R(4,-2 k, 6 k ; n)$. Thus

$$
\phi_{k}(n)=\frac{1}{2}(R(1,-k, 6 k ; n)-R(k+1,5 k, 6 k ; n)) .
$$

Clearly $(1,-k, 6 k) \sim\left(1, \delta_{k},\left(24 k-k^{2}+\delta_{k}\right) / 4\right)$ and $(k+1,5 k, 6 k) \sim(k+1$, $k-4,4) \sim(4,4-k, k+1)$. If $9 \leq k \leq 12$, then $(4,4-k, k+1) \sim(4,12-k, 9)$. Also, $(4,3,2) \sim(2,-3,4) \sim(2,1,3)$. Now combining the above we get the desired result.

Corollary 2.1. Let $n \in \mathbb{N}, 2 \mid n$ and $m \in\{1,2,3,4,5,6\}$. Then

$$
R(1,0, m(12-m) ; n)=R(4,4-2 m, 2 m+1 ; n) .
$$

Proof. Note that $\phi_{2 m}(n)=0$ by Definition 2.3. Putting $k=2 m$ in Theorem 2.2 gives the result.

Corollary 2.1 can also be deduced from [KW2, Theorem 1]. For $m=$ 1, 2, 3, 4, 5 see Corollaries 1, 4, 5, 6, 8 in [KW2] respectively.

Let $\tau$ be the Ramanujan tau function defined by

$$
q \prod_{n=1}^{\infty}\left(1-q^{n}\right)^{24}=\sum_{n=1}^{\infty} \tau(n) q^{n} \quad(|q|<1)
$$

Then we have

Corollary 2.2. For any positive integer $n$ we have

$$
\tau(n) \equiv\left\{\begin{aligned}
0(\bmod 23) & \text { if there is a prime } p \text { such that }\left(\frac{p}{23}\right)=-1 \text { and } \\
2 \nmid \operatorname{ord}_{p} n, \text { or } p=2 x^{2}+x y+3 y^{2} \text { and } & \begin{array}{r}
3 \mid \operatorname{ord}_{p} n-2, \\
(-1)^{\mu}
\end{array} \prod_{p=x^{2}+x y+6 y^{2} \neq 23}\left(1+\operatorname{ord}_{p} n\right)(\bmod 23) \quad \text { otherwise, }
\end{aligned}\right.
$$

where

$$
\mu=\sum_{\substack{p=2 x^{2}+x y+3 y^{2} \\ \operatorname{ord}_{p} n \equiv 1(\bmod 3)}} 1
$$

and $p$ runs over all primes. 
Proof. Euler's identity states that (see for example [HW, Theorem 353, p. 284])

$$
\sum_{n=-\infty}^{\infty}(-1)^{n} q^{\left(3 n^{2}-n\right) / 2}=\prod_{n=1}^{\infty}\left(1-q^{n}\right) \quad(|q|<1)
$$

So

$$
\begin{aligned}
\frac{\sum_{n=1}^{\infty} \tau(n) q^{n}}{\sum_{n=1}^{\infty} \phi_{1}(n) q^{n}} & =\frac{q \prod_{n=1}^{\infty}\left(1-q^{n}\right)^{24}}{q \prod_{n=1}^{\infty}\left(1-q^{n}\right) \prod_{n=1}^{\infty}\left(1-q^{23 n}\right)}=\frac{\prod_{n=1}^{\infty}\left(1-q^{n}\right)^{23}}{\prod_{n=1}^{\infty}\left(1-q^{23 n}\right)} \\
& =\prod_{n=1}^{\infty}\left(1+\left(\sum_{k=1}^{22}\left(\begin{array}{c}
23 \\
k
\end{array}\right)(-1)^{k} q^{k n}\right)\left(1-q^{23 n}\right)^{-1}\right) \\
& =1+23 \sum_{n=1}^{\infty} a_{n} q^{n} \quad\left(a_{n} \in \mathbb{Z}\right)
\end{aligned}
$$

and hence applying Theorem 2.2 we get

$$
\tau(n) \equiv \phi_{1}(n)=\frac{1}{2}(R(1,1,6 ; n)-R(2,1,3 ; n))=F([2,1,3], n)(\bmod 23) .
$$

Observe that $H(-23)=\{[1,1,6],[2,1,3],[2,-1,3]\}$. Then applying $[\mathrm{SW}$, Theorem 10.2(i)] we obtain the result.

We remark that Corollary 2.2 generalizes the known result $23 \mid \tau(n)$ for those positive integers $n$ such that $\left(\frac{n}{23}\right)=-1$ (see for example [BO]).

TheOREM 2.3. For $k=1, \ldots, 8$ and $n \in \mathbb{N}$ we have $f_{2 k}(1,4 ; n)+f_{2 k}(3,4 ; n)=\frac{1}{2}(R(1,0, k(16-k) ; n)-R(4,4-2 k, 3 k+1 ; n))$.

Proof. By Proposition 2.1 we have

$$
\begin{aligned}
f_{2 k}(1,4 ; n)+ & f_{2 k}(3,4 ; n) \\
= & \sum_{\substack{x, y \in \mathbb{Z} \\
x^{2}-2 k x y+16 k y^{2}=n \\
x \equiv 1,3(\bmod 8)}} 1-\sum_{\substack{x, y \in \mathbb{Z} \\
(3 k+1) x^{2}+14 k x y+16 k y^{2}=n \\
x \equiv 1,3(\bmod 8)}} 1 \\
= & \frac{1}{2}\left(\sum_{\substack{x, y \in \mathbb{Z}, 2 \nmid x \\
x^{2}-2 k x y+16 k y^{2}=n}} 1-\sum_{\substack{x, y \in \mathbb{Z}, 2 \nmid x \\
(3 k+1) x^{2}+14 k x y+16 k y^{2}=n}} 1\right) \\
= & \frac{1}{2}\{(R(1,-2 k, 16 k ; n)-R(4,-4 k, 16 k ; n)) \\
& -(R(3 k+1,14 k, 16 k ; n)-R(12 k+4,28 k, 16 k ; n))\} .
\end{aligned}
$$

Note that $(12 k+4,28 k, 16 k) \sim(16 k,-28 k, 12 k+4) \sim(16 k, 4 k, 4) \sim$ $(4,-4 k, 16 k),(1,-2 k, 16 k) \sim(1,0, k(16-k))$ and $(3 k+1,14 k, 16 k) \sim$ $(3 k+1,2 k-4,4) \sim(4,4-2 k, 3 k+1)$. We then obtain the result. 
Theorem 2.4. Let $k \in\{1, \ldots, 20\}, \delta_{k}=\left(1-(-1)^{k}\right) / 2, n \in \mathbb{N}$. Then

$$
\begin{aligned}
f_{k}(1,5 ; n) & +f_{k}(3,5 ; n) \\
& =\frac{1}{2}\left(R\left(1, \delta_{k},\left(40 k-k^{2}+\delta_{k}\right) / 4 ; n\right)-R(4,4-k, 2 k+1 ; n)\right) .
\end{aligned}
$$

Proof. By Proposition 2.1 we have

$$
\begin{aligned}
f_{k}(1,5 ; n) & +f_{k}(3,5 ; n) \\
= & \sum_{\substack{x, y \in \mathbb{Z} \\
x^{2}-k x y+10 k y^{2}=n \\
x \equiv 1,3(\bmod 10)}} 1-\sum_{\substack{x, y \in \mathbb{Z} \\
(2 k+1) x^{2}+9 k x y+10 k y^{2}=n \\
x \equiv 1,3(\bmod 10)}} 1 \\
= & \frac{1}{2}\left(\sum_{\substack{x, y \in \mathbb{Z}, 2 \nmid x \\
x^{2}-k x y+10 k y^{2}=n}} 1-\sum_{\substack{x, y \in \mathbb{Z}, x \equiv 5(\bmod 10) \\
x^{2}-k x y+10 k y^{2}=n}} 1\right) \\
& -\frac{1}{2}\left(\sum_{\substack{x, y \in \mathbb{Z}, 2 \nmid x \\
(2 k+1) x^{2}+9 k x y+10 k y^{2}=n}} \sum_{(2 k+1) x^{2}+9 k x y+10 k y^{2}=n} 1\right) \\
& -\frac{1}{2}\{(R(1,-k, 10 k ; n)-R(4,-2 k, 10 k ; n)) \\
& -(R(25,-5 k, 10 k ; n)-R(100,-10 k, 10 k ; n)) \\
& +(R(2 k+1,9 k, 10 k ; n)-R(8 k+4,18 k, 10 k ; n))
\end{aligned}
$$

Observe that $(8 k+4,18 k, 10 k) \sim(8 k+4,2 k-8,4) \sim(4,8-2 k, 8 k+4) \sim$ $(4,-2 k, 10 k),(50 k+25,45 k, 10 k) \sim(10 k,-45 k, 50 k+25) \sim(10 k,-5 k, 25)$ $\sim(25,5 k, 10 k)$ and $(200 k+100,90 k, 10 k) \sim(10 k,-90 k, 200 k+100) \sim$ $(10 k, 10 k, 100) \sim(100,-10 k, 10 k)$. We then obtain

$$
f_{k}(1,5 ; n)+f_{k}(3,5 ; n)=\frac{1}{2}\{R(1,-k, 10 k ; n)-R(2 k+1,9 k, 10 k ; n)\} .
$$

Since $(1,-k, 10 k) \sim\left(1, \delta_{k},\left(40 k-k^{2}+\delta_{k}\right) / 4\right)$ and $(2 k+1,9 k, 10 k) \sim(2 k+1$, $k-4,4) \sim(4,4-k, 2 k+1)$, we obtain the desired result.

Corollary 2.3. If $n$ is even and $m \in\{1, \ldots, 10\}$, then

$$
R(1,0, m(20-m) ; n)=R(4,4-2 m, 4 m+1 ; n) .
$$

Proof. Note that $f_{2 m}(1,5 ; n)=f_{2 m}(3,5 ; n)=0$ by Definition 2.1. Taking $k=2 m$ in Theorem 2.4 yields the result.

The case $m=1$ of Corollary 2.3 has been given in [KW2, Corollary 3].

Corollary 2.4. For $n \in \mathbb{N}$ we have

(i) $f_{2}(1,4 ; n)+f_{2}(3,4 ; n)=\psi_{3}(n)$,

(ii) $f_{4}(1,5 ; n)+f_{4}(3,5 ; n)=\phi_{12}(n)$,

(iii) $f_{8}(1,5 ; n)+f_{8}(3,5 ; n)=f_{16}(1,4 ; n)+f_{16}(3,4 ; n)$. 
Proof. From Theorems 2.1-2.4 we see that

$$
\begin{aligned}
& f_{2}(1,4 ; n)+f_{2}(3,4 ; n)=\frac{1}{2}(R(1,0,15 ; n)-R(4,2,4 ; n))=\psi_{3}(n), \\
& f_{4}(1,5 ; n)+f_{4}(3,5 ; n)=\frac{1}{2}(R(1,0,36 ; n)-R(4,0,9 ; n))=\phi_{12}(n)
\end{aligned}
$$

and

$$
\begin{aligned}
& f_{8}(1,5 ; n)+f_{8}(3,5 ; n) \\
& \quad=\frac{1}{2}(R(1,0,64 ; n)-R(4,4,17 ; n))=\frac{1}{2}(R(1,0,64 ; n)-R(4,-12,25 ; n)) \\
& \quad=f_{16}(1,4 ; n)+f_{16}(3,4 ; n) .
\end{aligned}
$$

So the corollary is proved.

THEOREM 2.5. Let $p$ be an odd prime. Let $r$ be odd with $p \nmid r$ and $n \in \mathbb{N}$. Then

$$
f_{4 p}(r, p ; n)= \begin{cases}\frac{1}{2}\left(R\left(1,0,4 p^{2} ; n\right)-R\left(4,0, p^{2} ; n\right)\right) & \text { if } n \equiv r^{2}(\bmod 4 p), \\ 0 & \text { otherwise. }\end{cases}
$$

Proof. From Proposition 2.1 we know that

$$
f_{4 p}(r, p ; n)=\sum_{\substack{x, y \in \mathbb{Z} \\ x^{2}-4 p x y+8 p^{2} y^{2}=n \\ x \equiv r(\bmod 2 p)}} 1-\sum_{\substack{x, y \in \mathbb{Z} \\(1+2 p(p-1)) x^{2}+4 p(2 p-1) x y+8 p^{2} y^{2}=n \\ x \equiv r(\bmod 2 p)}}
$$

If $n \not \equiv r^{2}(\bmod 4 p)$, then clearly $f_{4 p}(r, p ; n)=0$ by Definition 2.1. If $n \equiv r^{2}$ $(\bmod 4 p)$, then $x^{2} \equiv n(\bmod 4 p)$ if and only if $x \equiv \pm r(\bmod 2 p)$. Thus, by the above we obtain

$$
f_{4 p}(r, p ; n)=\frac{1}{2} R\left(1,-4 p, 8 p^{2} ; n\right)-\frac{1}{2} R\left(2 p^{2}-2 p+1,4 p(2 p-1), 8 p^{2} ; n\right) .
$$

Since $\left(1,-4 p, 8 p^{2}\right) \sim\left(1,0,4 p^{2}\right)$ and $\left(2 p^{2}-2 p+1,4 p(2 p-1), 8 p^{2}\right) \sim\left(2 p^{2}-\right.$ $2 p+1,4 p-4,4) \sim\left(4,4-4 p, 2 p^{2}-2 p+1\right) \sim\left(4,0, p^{2}\right)$, we obtain the result.

3. The Euler product for $\sum_{n=1}^{\infty} \frac{\Delta(n, d)}{n^{s}}$. Let $d$ be a discriminant with conductor $f$ and $d_{0}=d / f^{2}$. In view of [SW, Lemma 3.5] we introduce

$$
C(d)=f \prod_{p \mid f}\left(1-\frac{1}{p}\left(\frac{d_{0}}{p}\right)\right)= \begin{cases}\frac{h(d) w\left(d_{0}\right)}{h\left(d_{0}\right) w(d)} & \text { if } d<0, \\ \frac{h(d) \log \varepsilon(d)}{h\left(d_{0}\right) \log \varepsilon\left(d_{0}\right)} & \text { if } d>0,\end{cases}
$$

where $p$ runs over all distinct prime divisors of $f$. If $f$ is a prime, then clearly $C(d)=f-\left(\frac{d_{0}}{p}\right)$.

Definition 3.1. Let $d$ be a discriminant with conductor $f$. Let $d_{0}=$ $d / f^{2}$ and $n \in \mathbb{N}$. Then we define

$$
\delta(n, d)=\sum_{m \mid n}\left(\frac{d}{m}\right)
$$


and

$$
\Delta(n, d)= \begin{cases}\delta(n, d) & \text { if } f \nmid n, \\ -C(d)\left(\frac{d_{0}}{f}\right)^{\operatorname{ord}_{f} n-1} \delta(n, d) & \text { if } f \mid n .\end{cases}
$$

LEMma 3.1 ([SW, Lemma 4.1]). Let $d$ be a discriminant and $n \in \mathbb{N}$. Then $\delta(n, d)$ is a multiplicative function of $n$. Moreover,

$$
\delta(n, d)=\prod_{\left(\frac{d}{p}\right)=-1} \frac{1+(-1)^{\operatorname{ord}_{p} n}}{2} \prod_{\left(\frac{d}{p}\right)=1}\left(1+\operatorname{ord}_{p} n\right)
$$

and

$$
\sum_{n=1}^{\infty} \frac{\delta(n, d)}{n^{s}}=\prod_{p} \frac{1}{\left(1-p^{-s}\right)\left(1-\left(\frac{d}{p}\right) p^{-s}\right)} \quad(\operatorname{Re}(s)>1) .
$$

From Definition 3.1 and Lemma 3.1 we have

Lemma 3.2. Let $d$ be a discriminant with conductor $f$. Let $s \in \mathbb{C}$ with $\operatorname{Re}(s)>1$. Set $d_{0}=d / f^{2}$. If $f$ is a prime, then $\Delta(n, d)$ is a multiplicative function of $n \in \mathbb{N}$ and

$$
\sum_{n=1}^{\infty} \frac{\Delta(n, d)}{n^{s}}=\left(1-\frac{C(d) f^{-s}}{1-\left(\frac{d_{0}}{f}\right) f^{-s}}\right) \prod_{p \neq f} \frac{1}{\left(1-p^{-s}\right)\left(1-\left(\frac{d_{0}}{p}\right) p^{-s}\right)} .
$$

LEMMA 3.3. Let $d$ be a discriminant such that $h(d)=1$ and the conductor $f$ is a prime. Set $d_{0}=d / f^{2}$ and $\delta_{k}=\left(1-(-1)^{k}\right) / 2$ for $k \in \mathbb{Z}$. For $n \in \mathbb{N}$ we have

$$
\Delta(n, d) \quad \begin{cases}\frac{1}{2}\left(R\left(1, \delta_{d}, \frac{-d+\delta_{d}}{4} ; n\right)-R\left(f, \delta_{d_{0}} f, \frac{-d_{0}+\delta_{d_{0}}}{4} f ; n\right)\right) & \text { if } d<0, \\ R\left(1, \delta_{d}, \frac{-d+\delta_{d}}{4} ; n\right)-C(d) R\left(f, \delta_{d_{0}} f, \frac{-d_{0}+\delta_{d_{0}}}{4} f ; n\right) & \text { if } d>0 .\end{cases}
$$

Proof. Let $N(n, d)=\sum_{K \in H(d)} R(K, n)$. From [SW, Theorem 4.1] we know that

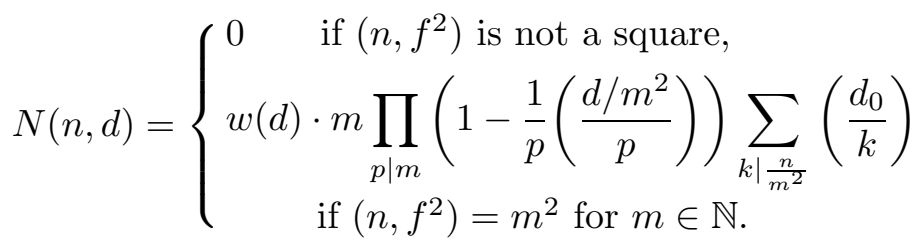


Observing that $h(d)=1$ and $f$ is a prime, by (3.2) we have

$$
R\left(1, \delta_{d},\left(-d+\delta_{d}\right) / 4 ; n\right)= \begin{cases}0 & \text { if } f \| n, \\ w(d) \sum_{k \mid n}\left(\frac{d_{0}}{k}\right) & \text { if } f \nmid n, \\ w(d) C(d) \sum_{k \mid \frac{n}{f^{2}}}\left(\frac{d_{0}}{k}\right) & \text { if } f^{2} \mid n .\end{cases}
$$

Since $h\left(d_{0}\right) \mid h(d)$ (see [SW, Remark 2.2]) we see that $h\left(d_{0}\right)=1$. If $f \nmid n$, clearly $R\left(f, \delta_{d_{0}} f,\left(-d_{0}+\delta_{d_{0}}\right) f / 4 ; n\right)=0$. If $f \mid n$, by (3.2), [SW, Remark 3.1] and the fact that $f\left(d_{0}\right)=1$ we have

$$
\begin{aligned}
R\left(f, \delta_{d_{0}} f,\left(-d_{0}+\delta_{d_{0}}\right) f / 4 ; n\right) & =R\left(1, \delta_{d_{0}},\left(-d_{0}+\delta_{d_{0}}\right) / 4 ; n / f\right) \\
& =w\left(d_{0}\right) \sum_{k \mid \frac{n}{f}}\left(\frac{d_{0}}{k}\right) .
\end{aligned}
$$

Thus

$$
\begin{aligned}
& \frac{1}{w(d)}\left(R\left(1, \delta_{d}, \frac{-d+\delta_{d}}{4} ; n\right)-\frac{C(d) w(d)}{w\left(d_{0}\right)} R\left(f, \delta_{d_{0}} f, \frac{-d_{0}+\delta_{d_{0}}}{4} f ; n\right)\right) \\
& =\left\{\begin{array}{lc}
\sum_{k \mid n}\left(\frac{d_{0}}{k}\right)-0 & \text { if } f \nmid n, \\
0-C(d) \sum_{k \mid \frac{n}{f}}\left(\frac{d_{0}}{k}\right)=-C(d) \sum_{k \mid n, f \nmid k}\left(\frac{d_{0}}{k}\right) & \text { if } f \| n, \\
-C(d) \sum_{k \mid \frac{n}{f}, k \nmid \frac{n}{f^{2}}}\left(\frac{d_{0}}{k}\right)=-C(d) \sum_{k \mid n, f \nmid k}\left(\frac{d_{0}}{\left.k f^{\operatorname{ord}_{f} n-1}\right)}\right) & \text { if } f^{2} \mid n .
\end{array}\right.
\end{aligned}
$$

To complete the proof, we note that

$$
\sum_{k \mid n, f \nmid k}\left(\frac{d_{0}}{k}\right)=\sum_{k \mid n}\left(\frac{d_{0} f^{2}}{k}\right)=\delta(n, d)
$$

and

$$
\frac{C(d) w(d)}{w\left(d_{0}\right)}= \begin{cases}1 & \text { if } d<0 \\ C(d) & \text { if } d>0\end{cases}
$$

If $q \equiv 1(\bmod 4)$ is a prime such that $h(4 q)=1$, then $h(q)=1$ by [SW, Remark 2.2]. From (3.1) we find $C(4 q)=2\left(1-\frac{1}{2}\left(\frac{q}{2}\right)\right)=2-(-1)^{(q-1) / 4}$. Now applying Lemmas 3.2 and 3.3 in the cases $d=-12,-16,-27,-28,4 q$ we get 
Theorem 3.1. Let $s \in \mathbb{C}$ with $\operatorname{Re}(s)>1$. Then

(a) $\sum_{n=1}^{\infty} \frac{\frac{1}{2}(R(1,0,3 ; n)-R(2,2,2 ; n))}{n^{s}}$

$=\frac{1-2^{1-s}}{1+2^{-s}} \cdot \frac{1}{1-3^{-s}} \prod_{p \equiv 5(\bmod 6)} \frac{1}{1-p^{-2 s}} \prod_{p \equiv 1(\bmod 6)} \frac{1}{\left(1-p^{-s}\right)^{2}}$,

(b) $\sum_{n=1}^{\infty} \frac{\frac{1}{2}(R(1,0,4 ; n)-R(2,0,2 ; n))}{n^{s}}$

$=\left(1-2^{1-s}\right) \prod_{p \equiv 3(\bmod 4)} \frac{1}{1-p^{-2 s}} \prod_{p \equiv 1(\bmod 4)} \frac{1}{\left(1-p^{-s}\right)^{2}}$,

(c) $\sum_{n=1}^{\infty} \frac{\frac{1}{2}(R(1,1,7 ; n)-R(3,3,3 ; n))}{n^{s}}$

$=\left(1-3^{1-s}\right) \prod_{p \equiv 2(\bmod 3)} \frac{1}{1-p^{-2 s}} \prod_{p \equiv 1(\bmod 3)} \frac{1}{\left(1-p^{-s}\right)^{2}}$,

(d) $\sum_{n=1}^{\infty} \frac{\frac{1}{2}(R(1,0,7 ; n)-R(2,2,4 ; n))}{n^{s}}$

$=\frac{1-2^{1-s}}{1-2^{-s}} \cdot \frac{1}{1-7^{-s}} \prod_{p \equiv 3,5,6(\bmod 7)} \frac{1}{1-p^{-2 s}} \prod_{\substack{p \equiv 1,2,4(\bmod 7) \\ p \neq 2}} \frac{1}{\left(1-p^{-s}\right)^{2}}$.

(e) If $q \equiv 1(\bmod 4)$ is a prime such that $h(4 q)=1$ (for example $q=$ $5,13,17,29,41,53,61,73,89,97,109,113, \ldots)$, then

$$
\begin{aligned}
& \sum_{n=1}^{\infty} \frac{R(1,0,-q ; n)-\left(2-(-1)^{(q-1) / 4}\right) R(2,2,(1-q) / 2 ; n)}{n^{s}} \\
& =\frac{1-2^{1-s}}{1-(-1)^{(q-1) / 4} 2^{-s}} \prod_{p>2} \frac{1}{\left(1-p^{-s}\right)\left(1-\left(\frac{q}{p}\right) p^{-s}\right)} .
\end{aligned}
$$

Theorem 3.2. For $s \in \mathbb{C}$ with $\operatorname{Re}(s)>1$ we have

$$
\begin{aligned}
\sum_{n=1}^{\infty} \frac{\psi_{1}(n)}{n^{s}}= & \left(2-\frac{1}{1-2^{-s}}\right) \cdot \frac{1}{1-7^{-s}} \prod_{p \equiv 3,5,6(\bmod 7)} \frac{1}{1-p^{-2 s}} \\
& \times \prod_{\substack{p \equiv 1,2,4(\bmod 7) \\
p \neq 2}} \frac{1}{\left(1-p^{-s}\right)^{2}}
\end{aligned}
$$


and

$$
\begin{aligned}
\sum_{n=1}^{\infty} \frac{(-1)^{n-1} \psi_{1}(n)}{n^{s}}= & \frac{1}{\left(1-2^{-s}\right)\left(1-7^{-s}\right)} \prod_{p \equiv 3,5,6(\bmod 7)} \frac{1}{1-p^{-2 s}} \\
& \times \prod_{\substack{p \equiv 1,2,4(\bmod 7) \\
p \neq 2}} \frac{1}{\left(1-p^{-s}\right)^{2}} .
\end{aligned}
$$

Proof. From Theorem 2.1 we know that

$$
\psi_{1}(n)=\frac{1}{2}(R(1,0,7 ; n)-R(2,2,4 ; n)) .
$$

Using Lemma 3.3 with $d=-28$ we see that

$$
\begin{aligned}
(-1)^{n-1} \psi_{1}(n) & =(-1)^{n-1}(R(1,0,7 ; n)-R(2,2,4 ; n)) / 2 \\
& =(-1)^{n-1} \Delta(n,-28)=\delta(n,-28) .
\end{aligned}
$$

Thus applying Lemma 3.1 and Theorem 3.1 we obtain the result.

\section{Values of $\psi_{k}(n)$ and $\phi_{k}(n)$ and related identities}

TheOrem 4.1. For $n \in \mathbb{N}$ we have

$$
\begin{aligned}
& \psi_{1}(n)=(-1)^{n-1} \sum_{m \mid n}\left(\frac{-28}{m}\right)=(-1)^{n-1} \sum_{m \mid n, 2 \nmid m}\left(\frac{m}{7}\right), \\
& \psi_{2}(n)= \begin{cases}(-1)^{(n-1) / 2} \sum_{m \mid n}\left(\frac{m}{3}\right) & \text { if } 2 \nmid n, \\
0 & \text { if } 2 \mid n\end{cases}
\end{aligned}
$$

and

$$
\psi_{4}(n)= \begin{cases}(-1)^{(n-1) / 4} \sum_{m \mid n}\left(\frac{-1}{m}\right) & \text { if } n \equiv 1(\bmod 4), \\ 0 & \text { if } n \neq 1(\bmod 4) .\end{cases}
$$

Proof. From the proof of Theorem 3.1 we see that

$$
(-1)^{n-1} \psi_{1}(n)=\delta(n,-28)=\sum_{m \mid n}\left(\frac{-28}{m}\right)=\sum_{m \mid n, 2 \nmid m}\left(\frac{-7}{m}\right)=\sum_{m \mid n, 2 \nmid m}\left(\frac{m}{7}\right) .
$$

According to Theorem 2.1, [SW, Theorem 9.2] and Lemma 3.1 we have

$$
\begin{aligned}
\psi_{2}(n) & =\frac{1}{2}(R(1,0,12 ; n)-R(3,0,4 ; n)) \\
& = \begin{cases}(-1)^{(n-1) / 2} \sum_{m \mid n}\left(\frac{-3}{m}\right)=(-1)^{(n-1) / 2} \sum_{m \mid n}\left(\frac{m}{3}\right) & \text { if } 2 \nmid n, \\
0 & \text { if } 2 \mid n,\end{cases}
\end{aligned}
$$




$$
\begin{aligned}
\psi_{4}(n) & =\frac{1}{2}(R(1,0,16 ; n)-R(4,4,5 ; n)) \\
& = \begin{cases}\left(\frac{2}{n}\right) \sum_{m \mid n}\left(\frac{-1}{m}\right) & \text { if } 2 \nmid n, \\
0 & \text { if } 2 \mid n\end{cases} \\
& = \begin{cases}(-1)^{(n-1) / 4} \sum_{m \mid n}\left(\frac{-1}{m}\right) & \text { if } n \equiv 1(\bmod 4), \\
0 & \text { otherwise. }\end{cases}
\end{aligned}
$$

In the last step we note that if $n \equiv 3(\bmod 4)$, then

$$
\sum_{m \mid n}\left(\frac{-1}{m}\right)=\sum_{\substack{m \mid n \\ m<\sqrt{n}}}\left(\left(\frac{-1}{m}\right)+\left(\frac{-1}{n / m}\right)\right)=\sum_{\substack{m \mid n \\ m<\sqrt{n}}}\left(\frac{-1}{m}\right)\left(1+\left(\frac{-1}{n}\right)\right)=0 .
$$

This completes the proof.

Theorem 4.2. For $n \in \mathbb{N}$ let $n=3^{\alpha} n_{0}\left(3 \nmid n_{0}\right)$. Then

$$
\psi_{3}(n)=(-1)^{n-1} \frac{1+(-1)^{\alpha}\left(\frac{n_{0}}{3}\right)}{2} \sum_{m \mid n, 2 \nmid m}\left(\frac{m}{15}\right) \text {. }
$$

Proof. By Theorem 2.1 we have

$$
\begin{aligned}
\psi_{3}(n) & =\frac{1}{2}(R(1,0,15 ; n)-R(4,-2,4 ; n)) \\
& = \begin{cases}\frac{1}{2} R(1,0,15 ; n) & \text { if } 2 \nmid n, \\
\frac{1}{2}(R(1,0,15 ; n)-R(2,1,2 ; n / 2)) & \text { if } 2 \mid n .\end{cases}
\end{aligned}
$$

Now we consider the following three cases.

CAsE 1: $2 \nmid n$. By the above, [SW, Theorem 9.3] and Lemma 3.1,

$$
\begin{aligned}
\psi_{3}(n) & =\frac{1}{2} R(1,0,15 ; n)=\frac{1+(-1)^{\alpha}\left(\frac{n_{0}}{3}\right)}{2} \sum_{m \mid n}\left(\frac{-15}{m}\right) \\
& =\frac{1+(-1)^{\alpha}\left(\frac{n_{0}}{3}\right)}{2} \sum_{m \mid n, 2 \nmid m}\left(\frac{m}{15}\right) .
\end{aligned}
$$

CASE 2: 2\|n. By [SW, Theorem 9.3] and Lemma 3.1, $R(1,0,15 ; n)=0$ and

$$
\begin{aligned}
R(2,1,2 ; n / 2) & =\left(1-(-1)^{\alpha}\left(\frac{n_{0} / 2}{3}\right)\right) \sum_{m \mid \frac{n}{2}}\left(\frac{-15}{m}\right) \\
& =\left(1+(-1)^{\alpha}\left(\frac{n_{0}}{3}\right)\right) \sum_{m \mid \frac{n}{2}}\left(\frac{m}{15}\right) .
\end{aligned}
$$


Thus

$$
\begin{aligned}
2 \psi_{3}(n) & =R(1,0,15 ; n)-R(2,1,2 ; n / 2) \\
& =-\left(1+(-1)^{\alpha}\left(\frac{n_{0}}{3}\right)\right) \sum_{m \mid \frac{n}{2}}\left(\frac{m}{15}\right) \\
& =-\left(1+(-1)^{\alpha}\left(\frac{n_{0}}{3}\right)\right) \sum_{m \mid n, 2 \nmid m}\left(\frac{m}{15}\right) .
\end{aligned}
$$

CASE 3: $4 \mid n$. From [SW, Theorem 9.3] and Lemma 3.1 we know that

$$
R(1,0,15 ; n)=\left(1+(-1)^{\alpha}\left(\frac{n_{0}}{3}\right)\right) \sum_{m \mid \frac{n}{4}}\left(\frac{m}{15}\right)
$$

and

$$
R(2,1,2 ; n / 2)=\left(1+(-1)^{\alpha}\left(\frac{n_{0}}{3}\right)\right) \sum_{m \mid \frac{n}{2}}\left(\frac{m}{15}\right)
$$

Thus

$\psi_{3}(n)=\frac{1}{2}(R(1,0,15 ; n)-R(2,1,2 ; n / 2))=-\frac{1+(-1)^{\alpha}\left(\frac{n_{0}}{3}\right)}{2} \sum_{m \mid \frac{n}{2}, m \nmid \frac{n}{4}}\left(\frac{m}{15}\right)$.

Observing that

$$
\sum_{m \mid \frac{n}{2}, m \nmid \frac{n}{4}}\left(\frac{m}{15}\right)=\sum_{m \mid n, 2 \nmid m}\left(\frac{2^{\mathrm{ord}_{2} n-1} m}{15}\right)=\sum_{m \mid n, 2 \nmid m}\left(\frac{m}{15}\right)
$$

we then get the desired result.

Summarizing the above we prove the following theorem.

TheOREM 4.3. Let $|q|<1$ and $\psi(q)=\sum_{n=0}^{\infty} q^{n(n+1) / 2}$. Then

(i) $q \psi(q) \psi\left(q^{7}\right)=\sum_{n=1}^{\infty}\left(\sum_{m \mid n, 2 \nmid m}\left(\frac{m}{7}\right)\right) q^{n}$.

(ii) $\psi(q) \psi\left(q^{3}\right)=\sum_{n=0}^{\infty}\left(\sum_{m \mid 2 n+1}\left(\frac{m}{3}\right)\right) q^{n}$.

(iii) $\psi^{2}(q)=\sum_{n=0}^{\infty}\left(\sum_{m \mid 4 n+1}(-1)^{(m-1) / 2}\right) q^{n}$.

(iv) $\psi^{4}(q)=\sum_{n=0}^{\infty}\left(\sum_{d \mid 2 n+1} d\right) q^{n}$. 
Proof. From Definition 2.2 we obtain

$$
q \psi(q) \psi\left(q^{7}\right)=\sum_{n=1}^{\infty}(-1)^{n-1} \psi_{1}(n) q^{n}
$$

Then, applying Theorem 4.1, we obtain (i). From Definition 2.2 and Theorem 4.1 we have

$$
q \psi\left(-q^{2}\right) \psi\left(-q^{6}\right)=\sum_{n=1}^{\infty} \psi_{2}(n) q^{n}=\sum_{n=0}^{\infty}(-1)^{n}\left(\sum_{m \mid 2 n+1}\left(\frac{m}{3}\right)\right) q^{2 n+1} .
$$

Thus

$$
\psi(-q) \psi\left(-q^{3}\right)=\sum_{n=0}^{\infty}(-1)^{n}\left(\sum_{m \mid 2 n+1}\left(\frac{m}{3}\right)\right) q^{n} .
$$

Replacing $q$ by $-q$ we then obtain (ii).

We now consider (iii). It follows from Definition 2.2 and Theorem 4.1 that

$$
q \psi^{2}\left(-q^{4}\right)=\sum_{n=1}^{\infty} \psi_{4}(n) q^{n}=\sum_{n=0}^{\infty}(-1)^{n}\left(\sum_{m \mid 4 n+1}\left(\frac{-1}{m}\right)\right) q^{4 n+1},
$$

from which (iii) follows.

Finally we consider (iv). Let $r_{s}(n)$ denote the number of ways in which $n$ can be represented as a sum of $s$ squares. It is well known that (cf. [IR, pp. 279, 282], [HW, pp. 242, 314])

$$
r_{2}(n)=4 \sum_{d \mid n, 2 \nmid d}(-1)^{(d-1) / 2} \text { and } \quad r_{4}(n)= \begin{cases}8 \sum_{d \mid n} d & \text { if } 2 \nmid n, \\ 24 \sum_{d \mid n, 2 \nmid d} d & \text { if } 2 \mid n .\end{cases}
$$

Set $\psi^{4}(q)=\sum_{n=0}^{\infty} c_{n} q^{n}$. From (iii) and the formula for $r_{2}(n)$ we see that

$$
16 \psi^{4}(q)=\left(4 \psi^{2}(q)\right)^{2}=\left(\sum_{m=0}^{\infty} r_{2}(4 m+1) q^{m}\right)^{2} .
$$

Thus, using the fact that $r_{2}(4 m+3)=0$ we derive that

$$
\begin{aligned}
16 c_{n} & =\sum_{\substack{k+m=4 n+2 \\
k, m \equiv 1(\bmod 4)}} r_{2}(k) r_{2}(m)=\sum_{\substack{1 \leq k \leq 4 n+2 \\
k \equiv 1(\bmod 4)}} r_{2}(k) r_{2}(4 n+2-k) \\
& =\sum_{\substack{1 \leq k \leq 4 n+2 \\
k \equiv 1(\bmod 2)}} r_{2}(k) r_{2}(4 n+2-k) \\
& =\sum_{0 \leq k \leq 4 n+2} r_{2}(k) r_{2}(4 n+2-k)-\sum_{0 \leq m \leq 2 n+1} r_{2}(2 m) r_{2}(4 n+2-2 m)
\end{aligned}
$$




$$
\begin{aligned}
& =r_{4}(4 n+2)-\sum_{0 \leq m \leq 2 n+1} r_{2}(m) r_{2}(2 n+1-m) \\
& =r_{4}(4 n+2)-r_{4}(2 n+1)=24 \sum_{d \mid 4 n+2,2 \nmid d} d-8 \sum_{d \mid 2 n+1} d \\
& =16 \sum_{d \mid 2 n+1} d .
\end{aligned}
$$

So (iv) is true and hence the theorem is proved.

Corollary 4.1 (Ramanujan). If $|q|<1$, then

$q \psi(q) \psi\left(q^{7}\right)=\frac{q}{1-q}-\frac{q^{3}}{1-q^{3}}-\frac{q^{5}}{1-q^{5}}+\frac{q^{9}}{1-q^{9}}+\frac{q^{11}}{1-q^{11}}-\frac{q^{13}}{1-q^{13}}+\cdots$ where the cycle of coefficients is of length 14.

Proof. Since

$$
\sum_{m \mid n, 2 \nmid m}\left(\frac{m}{7}\right)=\sum_{\substack{m \mid n \\ m \equiv 1,9,11(\bmod 14)}} 1-\sum_{\substack{m \mid n \\ m \equiv 3,5,13(\bmod 14)}} 1,
$$

the result follows from Theorem 4.3(i).

REMARK 4.1. Corollary 4.1 was first found by S. Ramanujan. In [B, pp. 302-303], Berndt wrote: "The first two formulas (Corollary 4.1 is the first item in Entry 17.) are of extreme interest, since they appear to indicate that Ramanujan was acquainted with a theorem equivalent to the addition theorem for elliptic integrals of the second kind. Although it would appear to be very difficult to prove (i) without this addition theorem, it is apparently not found in the notebooks." In 1999, Williams ([W]) gave a proof of Theorem 4.3(i) and Corollary 4.1 without the use of elliptic integrals. Clearly we also prove the above result of Ramanujan without the addition theorem for elliptic integrals.

From Theorem 4.3(ii) one can easily deduce

Corollary 4.2 (Ramanujan ([B], [W, p. 378])). If $|q|<1$, then

$$
q \psi\left(q^{2}\right) \psi\left(q^{6}\right)=\frac{q}{1-q^{2}}-\frac{q^{5}}{1-q^{10}}+\frac{q^{7}}{1-q^{14}}-\frac{q^{11}}{1-q^{22}}+\cdots
$$

REMARK 4.2. By equating powers of $q^{n}$ in Theorem 4.3 we obtain

$$
\begin{aligned}
& \left|\left\{(x, y) \mid n-1=\frac{x(x+1)}{2}+7 \cdot \frac{y(y+1)}{2}, x, y \in \mathbb{N} \cup\{0\}\right\}\right|=\sum_{m \mid n, 2 \nmid m}\left(\frac{m}{7}\right), \\
& \left|\left\{(x, y) \mid n=\frac{x(x+1)}{2}+3 \cdot \frac{y(y+1)}{2}, x, y \in \mathbb{N} \cup\{0\}\right\}\right|=\sum_{m \mid 2 n+1}\left(\frac{m}{3}\right),
\end{aligned}
$$




$$
\begin{gathered}
\left|\left\{(x, y) \mid n=\frac{x(x+1)}{2}+\frac{y(y+1)}{2}, x, y \in \mathbb{N} \cup\{0\}\right\}\right|=\sum_{m \mid 4 n+1}(-1)^{(m-1) / 2}, \\
\mid\left\{(x, y, z, t) \mid n=\frac{x(x+1)}{2}+\frac{y(y+1)}{2}+\frac{z(z+1)}{2}+\frac{t(t+1)}{2},\right. \\
x, y, z, t \in \mathbb{N} \cup\{0\}\} \mid=\sum_{d \mid 2 n+1} d .
\end{gathered}
$$

The last two formulae give the number of representations of $n \in \mathbb{N}$ as the sum of two and four triangular numbers respectively. Proofs of these formulae have been given by Adiga $[\mathrm{A}]$ and Ono, Robins and Wahl [ORW]. The latter formula was known to Legendre [L]. From Definition 2.2, Theorem 4.2 and [W, (53)] we deduce that if $n=3^{\alpha} n_{0} \geq 2\left(3 \nmid n_{0}\right)$, then

$$
\begin{aligned}
\mid\{(x, y) \mid n-1=3 x(x+1) / 2+ & 5 y(y+1) / 2, x, y \in \mathbb{N} \cup\{0\}\} \mid \\
& =\frac{1}{2}\left(1+(-1)^{\alpha}\left(\frac{n_{0}}{3}\right)\right) \sum_{m \mid n, 2 \nmid m}\left(\frac{m}{15}\right)
\end{aligned}
$$

and

$$
\begin{aligned}
\mid\{(x, y) \mid n-2=x(x+1) / 2+ & 15 y(y+1) / 2, x, y \in \mathbb{N} \cup\{0\}\} \mid \\
= & \frac{1}{2}\left(1-(-1)^{\alpha}\left(\frac{n_{0}}{3}\right)\right) \sum_{m \mid n, 2 \nmid m}\left(\frac{m}{15}\right) .
\end{aligned}
$$

Using modular equations Ramanujan proved (see [B, p. 139])

$$
\begin{gathered}
\psi^{2}(q)=\sum_{k=0}^{\infty}(-1)^{k} q^{k(k+1)} \frac{1+q^{2 k+1}}{1-q^{2 k+1}}, \quad \psi^{4}\left(q^{2}\right)=\sum_{n=0}^{\infty} \frac{(2 n+1) q^{2 n+1}}{1-q^{4 n+2}}, \\
q \psi^{8}(q)=\sum_{n=0}^{\infty} \frac{n^{3} q^{n}}{1-q^{2 n}} \quad(|q|<1) .
\end{gathered}
$$

Theorem 4.4. Let $n \in \mathbb{N}$. Then

(i)

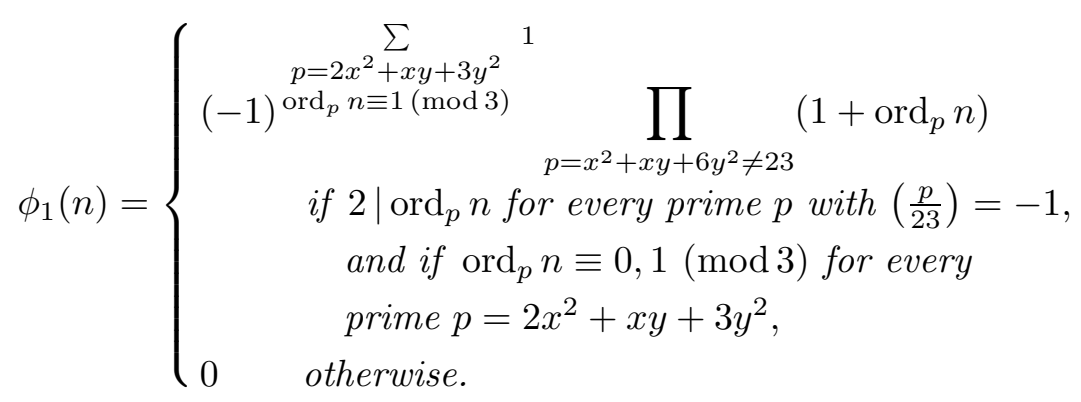


(ii) If $2 \mid n$, then $\phi_{2}(n)=0$. If $2 \nmid n$, then

$$
\phi_{2}(n)=\left\{\begin{array}{c}
\sum_{p=3 x^{2}+2 x y+4 y^{2}}{ }^{\operatorname{ord}_{p} n \equiv 1(\bmod 3)} \\
\text { if } 2 \mid \operatorname{ord}_{p} n \text { for every odd prime } p \equiv 2,6,7,8,10 \\
(\bmod 11), \text { and if } \operatorname{ord}_{p} n \equiv 0,1(\bmod 3) \text { for } \\
\text { every prime } p=3 x^{2}+2 x y+4 y^{2}, \\
\text { otherwise. }
\end{array}\right.
$$

(iii) If $2 \mid n$ or $3 \mid n$, then $\phi_{6}(n)=0$. If $2 \nmid n$ and $3 \nmid n$, then

$$
\phi_{6}(n)=\left\{\begin{array}{c}
\sum_{p=4 x^{2}+2 x y+7 y^{2}}^{1} \\
(-1)^{\operatorname{ord}_{p} n \equiv 1(\bmod 3)} \prod_{p=x^{2}+27 y^{2}}\left(1+\operatorname{ord}_{p} n\right) \\
\text { if } 2 \mid \operatorname{ord}_{p} n \text { for every prime } p \equiv 5(\bmod 6), \\
\text { and if } \operatorname{ord}_{p} n \equiv 0,1(\bmod 3) \text { for every } \\
\text { prime } p=4 x^{2}+2 x y+7 y^{2} \\
\text { otherwise. }
\end{array}\right.
$$

Proof. From Theorem 2.2 we know that

$$
\begin{array}{ll}
\phi_{1}(n)=\frac{1}{2}(R(1,1,6 ; n)-R(2,1,3 ; n))=F([2,1,3], n) & (d=-23), \\
\phi_{2}(n)=\frac{1}{2}(R(1,0,11 ; n)-R(3,2,4 ; n))=F([3,2,4], n) & (d=-44), \\
\phi_{6}(n)=\frac{1}{2}(R(1,0,27 ; n)-R(4,2,7 ; n))=F([4,2,7], n) & (d=-108) .
\end{array}
$$

Thus applying [SW, Theorem 10.2] we obtain the result.

Theorem 4.5. Let $n \in \mathbb{N}$. Then

$$
\phi_{3}(n)=\left\{\begin{array}{c}
(-1)^{\mu} \prod_{p \equiv 1,4,16(\bmod 21)}\left(1+\operatorname{ord}_{p} n\right) \\
\text { if } 3 \nmid n \text { and } 2 \mid \operatorname{ord}_{p} n \text { for every } \\
\text { prime } p \neq 1,4,7,16(\bmod 21) \\
\text { otherwise }
\end{array}\right.
$$

where

$$
\mu=\sum_{\substack{p \equiv 2,8,11(\bmod 21) \\
\operatorname{ord}_{p} n \equiv 2(\bmod 4)}} 1+\sum_{\begin{array}{c}
p=4 x^{2}+x y+4 y^{2} \\
\operatorname{ord}_{p} n \equiv 1(\bmod 2)
\end{array}} 1 .
$$


(ii)

$$
\phi_{4}(n)=\left\{\begin{array}{c}
(-1)^{\mu} \prod_{p \equiv 1,9(\bmod 20)}\left(1+\operatorname{ord}_{p} n\right) \\
\text { if } 2 \nmid n \text { and } 2 \mid \operatorname{ord}_{p} n \\
\text { for every prime } p \not \equiv 1,5,9(\bmod 20), \\
\text { otherwise, }
\end{array}\right.
$$

where

$$
\mu=\sum_{\substack{p \equiv 3,7(\bmod 20) \\
\operatorname{ord}_{p} n \equiv 2(\bmod 4)}} 1+\sum_{\begin{array}{c}
p=4 x^{2}+5 y^{2} \\
\operatorname{ord}_{p} n \equiv 1(\bmod 2)
\end{array}} 1 .
$$

(iii)

$$
\phi_{8}(n)=\left\{\begin{array}{c}
(-1)^{\mu} \prod_{p \equiv 1(\bmod 8)}\left(1+\operatorname{ord}_{p} n\right) \\
\text { if } 2 \nmid n \text { and } 2 \mid \operatorname{ord}_{p} n \\
\text { for every prime } p \not \equiv 1(\bmod 8), \\
\text { otherwise, }
\end{array}\right.
$$

where

$$
\mu=\sum_{\substack{p \equiv 3(\bmod 8) \\ \operatorname{ord}_{p} n \equiv 2(\bmod 4)}} 1+\sum_{\substack{p=4 x^{2}+4 x y+9 y^{2} \\ \operatorname{ord}_{p} n \equiv 1(\bmod 2)}} 1 .
$$

(iv)

$$
\phi_{12}(n)=\left\{\begin{array}{c}
(-1)^{\mu} \prod_{p \equiv 1(\bmod 12)}\left(1+\operatorname{ord}_{p} n\right) \\
\text { if }(n, 6)=1 \text { and } 2 \mid \operatorname{ord}_{p} n \\
\quad \text { for every prime } p \not \equiv 1(\bmod 12), \\
\text { otherwise, }
\end{array}\right.
$$

where

$$
\mu=\sum_{\substack{p \equiv 5(\bmod 12) \\
\operatorname{ord}_{p} n \equiv 2(\bmod 4)}} 1+\sum_{\begin{array}{c}
p=4 x^{2}+9 y^{2} \\
\operatorname{ord}_{p} n \equiv 1(\bmod 2)
\end{array}} 1 .
$$

Proof. From [SW, Proposition 11.1(i)] we know that $H(d)$ is a cyclic group of order 4 when $d \in\{-63,-80,-128,-144\}$. By Theorem 2.2 we have

$$
\begin{aligned}
\phi_{3}(n) & =\frac{1}{2}(R(1,1,16 ; n)-R(4,1,4 ; n))=F([2,1,8], n) & & (d=-63), \\
\phi_{4}(n) & =\frac{1}{2}(R(1,0,20 ; n)-R(4,0,5 ; n))=F([3,2,7], n) & & (d=-80), \\
\phi_{8}(n) & =\frac{1}{2}(R(1,0,32 ; n)-R(4,4,9 ; n))=F([3,2,11], n) & & (d=-128), \\
\phi_{12}(n) & =\frac{1}{2}(R(1,0,36 ; n)-R(4,0,9 ; n))=F([5,4,8], n) & & (d=-144) .
\end{aligned}
$$

For any prime $p$ it is clear that

$$
\begin{aligned}
& p \in R([2,1,8]) \Leftrightarrow p \equiv 2,8,11(\bmod 21), \\
& p \neq 7, p \in R([1,1,16]) \cup R([4,1,4]) \Leftrightarrow p \equiv 1,4,16(\bmod 21), \\
& p \in R([3,2,7]) \Leftrightarrow p \equiv 3,7(\bmod 20),
\end{aligned}
$$




$$
\begin{aligned}
& p \neq 2,5, p \in R([1,0,20]) \cup R([4,0,5]) \Leftrightarrow p \equiv 1,9(\bmod 20), \\
& p \in R([3,2,11]) \Leftrightarrow p \equiv 3(\bmod 8), \\
& p \neq 2, p \in R([1,0,32]) \cup R([4,4,9]) \Leftrightarrow p \equiv 1(\bmod 8), \\
& p \in R([5,4,8]) \Leftrightarrow p \equiv 5(\bmod 12), \\
& p \neq 2, p \in R([1,0,36]) \cup R([4,0,9]) \Leftrightarrow p \equiv 1(\bmod 12) .
\end{aligned}
$$

Thus applying [SW, Theorem 11.1] in the cases $d=-63,-80,-128,-144$ yields the result.

5. The Euler product for $L(M, s)(M \in H(d))$. Let $d$ be a discriminant. Suppose

$$
H(d)=\left\{A_{1}^{k_{1}} \cdots A_{r}^{k_{r}} \mid 0 \leq k_{1}<h_{1}, \ldots, 0 \leq k_{r}<h_{r}\right\}
$$

with $h_{1} \cdots h_{r}=h(d)$. For $n \in \mathbb{N}$ and $M=A_{1}^{m_{1}} \cdots A_{r}^{m_{r}} \in H(d)$, following [SW, Definition 7.1] we define

$$
\begin{aligned}
F(M, n)= & \frac{1}{w(d)} \sum_{\substack{0 \leq k_{1}<h_{1} \\
\cdots \\
0 \leq k_{r}<h_{r}}} \cos 2 \pi\left(\frac{k_{1} m_{1}}{h_{1}}+\cdots+\frac{k_{r} m_{r}}{h_{r}}\right) \\
& \times R\left(A_{1}^{k_{1}} \cdots A_{r}^{k_{r}}, n\right) .
\end{aligned}
$$

Let $N(n, d)=\sum_{M \in H(d)} R(M, n)$. Let $s \in \mathbb{C}$ be such that $\operatorname{Re}(s)>1$. From [SW, Theorem 4.1] and the same argument as in the proof of [HKW, Corollary 9.1] we know that for any $\varepsilon>0$ there exists a constant $C(\varepsilon)$ such that $N(n, d) \leq C(\varepsilon) n^{\varepsilon}$. Letting $\varepsilon \in(0, \operatorname{Re}(s)-1)$ we see that $\sum_{n=1}^{\infty} N(n, d) n^{-s}$ converges absolutely. Hence $\sum_{n=1}^{\infty} R(M, n) n^{-s}$ and $\sum_{n=1}^{\infty} F(M, n) n^{-s}$ converge absolutely since $R(M, n) \leq N(n, d)$ and $|F(M, n)| \leq N(n, d) / w(d)$. Using the same argument, for $p>1$ and $\operatorname{Re}(s)>1$ we see that $\sum_{t=1}^{\infty} F\left(M, p^{t}\right) p^{-s t}$ converges absolutely.

Definition 5.1. Let $d$ be a discriminant and $M \in H(d)$. Let $s \in \mathbb{C}$ be such that $\operatorname{Re}(s)>1$. Define

$$
Z(M, s)=\sum_{n=1}^{\infty} \frac{R(M, n)}{n^{s}} \quad \text { and } \quad L(M, s)=\sum_{n=1}^{\infty} \frac{F(M, n)}{n^{s}} .
$$

Then $Z(M, s)$ and $L(M, s)$ are analytic functions of $s$ in $\operatorname{Re}(s)>1$, and they can be continued analytically to the whole complex plane except for a simple pole at $s=1$.

Let $d$ be a discriminant and $M=[a, b, c] \in H(d)$. For $\operatorname{Re}(s)>1$ it is clear that

$$
Z(M, s)=\sum_{n=1}^{\infty} \frac{R(M, n)}{n^{s}}=\sum_{n=1}^{\infty} \frac{R(a, b, c ; n)}{n^{s}}
$$




$$
=\sum_{n=1}^{\infty} \sum_{\substack{\{x, y\} \text { is primary } \\ n=a x^{2}+b x y+c y^{2}}} \frac{1}{n^{s}}=\sum_{\{x, y\} \text { is primary }} \frac{1}{\left(a x^{2}+b x y+c y^{2}\right)^{s}} .
$$

Thus,

$$
Z(M, s)=\sum_{\substack{x, y \in \mathbb{Z} \\(x, y) \neq(0,0)}} \frac{1}{\left(a x^{2}+b x y+c y^{2}\right)^{s}} \quad \text { for } d<0
$$

and

$$
Z(M, s)=\sum_{\substack { x, y \in \mathbb{Z} \\
\begin{subarray}{c}{2 a x+(b-\sqrt{d}) y>0 \\
1 \leq\left|\frac{2 a x+(b+\sqrt{d}) y}{2 a x+(b-\sqrt{d}) y}\right|<\varepsilon(d)^{2}{ x , y \in \mathbb { Z } \\
\begin{subarray} { c } { 2 a x + ( b - \sqrt { d } ) y > 0 \\
1 \leq | \frac { 2 a x + ( b + \sqrt { d } ) y } { 2 a x + ( b - \sqrt { d } ) y } | < \varepsilon ( d ) ^ { 2 } } }\end{subarray}} \frac{1}{\left(a x^{2}+b x y+c y^{2}\right)^{s}} \quad \text { for } d>0
$$

For a negative discriminant $d$ and $M \in H(d)$, by (5.3) and a classical result due to $M$. Lerch (see $[\mathrm{D}],[\mathrm{ZW}],[\mathrm{SC}]$ ) we have the following functional equation for $Z(M, s)$ :

$$
\left(\frac{\sqrt{-d}}{2 \pi}\right)^{s} \Gamma(s) Z(M, s)=\left(\frac{\sqrt{-d}}{2 \pi}\right)^{1-s} \Gamma(1-s) Z(M, 1-s),
$$

where $\Gamma(s)$ is the Gamma function.

For a positive discriminant $d$ and $M \in H(d)$, we do not know if $Z(M, s)$ has a functional equation like (5.5).

TheOREM 5.1. Let $d$ be a discriminant. Let $M \in H(d)$ and $s \in \mathbb{C}$.

(i) If $\operatorname{Re}(s)>1$, then

$$
L(M, s)=\prod_{p}\left(1+\sum_{t=1}^{\infty} F\left(M, p^{t}\right) p^{-s t}\right) .
$$

(ii) If $d<0$ and $s \neq 0,1$, then

$$
\left(\frac{\sqrt{-d}}{2 \pi}\right)^{s} \Gamma(s) L(M, s)=\left(\frac{\sqrt{-d}}{2 \pi}\right)^{1-s} \Gamma(1-s) L(M, 1-s) .
$$

Proof. From [SW, Theorem 7.2] we know that $F(M, n)$ is a multiplicative function of $n \in \mathbb{N}$. By the previous argument, $L(M, s)$ and $\sum_{t=1}^{\infty} F\left(M, p^{t}\right) p^{-s t}$ converge absolutely if $\operatorname{Re}(s)>1$. Thus, for $m \in \mathbb{N}$ and $\operatorname{Re}(s)>1$ we have

$$
\begin{aligned}
\prod_{p \leq m}\left(1+\sum_{t=1}^{\infty} F\left(M, p^{t}\right) p^{-s t}\right) & =\sum_{n} F\left(M, \prod_{p \leq m} p^{\operatorname{ord}_{p} n}\right)\left(\prod_{p \leq m} p^{\operatorname{ord}_{p} n}\right)^{-s} \\
& =\sum_{n=1}^{m} \frac{F(M, n)}{n^{s}}+R_{m}(M, s)
\end{aligned}
$$


where the first sum is taken over all those positive integers $n$ whose prime divisors are less than $m$, and clearly

$$
\left|R_{m}(M, s)\right| \leq \sum_{n=m+1}^{\infty}\left|F(M, n) n^{-s}\right| .
$$

Since $\sum_{n=1}^{\infty} F(M, n) n^{-s}$ converges absolutely, we see that if $m \rightarrow \infty$, then $\sum_{n=m+1}^{\infty}\left|F(M, n) n^{-s}\right| \rightarrow 0$ and so $R_{m}(M, s) \rightarrow 0$. Therefore

converges and

$$
\prod_{p}\left(1+\sum_{t=1}^{\infty} F\left(M, p^{t}\right) p^{-s t}\right)
$$

$$
L(M, s)=\sum_{n=1}^{\infty} \frac{F(M, n)}{n^{s}}=\prod_{p}\left(1+\sum_{t=1}^{\infty} F\left(M, p^{t}\right) p^{-s t}\right) \quad \text { for } \operatorname{Re}(s)>1 .
$$

This proves (i).

Now we consider (ii). Suppose $H(d)$ is given by (5.1), $d<0$ and $M=$ $A_{1}^{m_{1}} \cdots A_{r}^{m_{r}}$. From (5.2) we see that

$$
\begin{aligned}
L(M, s) & =\frac{1}{w(d)} \sum_{\substack{0 \leq k_{1}<h_{1} \\
0 \leq k_{r}<h_{r} \\
0}} \cos 2 \pi\left(\frac{k_{1} m_{1}}{h_{1}}+\cdots+\frac{k_{r} m_{r}}{h_{r}}\right) \sum_{n=1}^{\infty} \frac{R\left(A_{1}^{k_{1}} \cdots A_{r}^{k_{r}}, n\right)}{n^{s}} \\
& =\frac{1}{w(d)} \sum_{\substack{0 \leq k_{1}<h_{1} \\
\cdots \\
0 \leq k_{r}<h_{r}}} \cos 2 \pi\left(\frac{k_{1} m_{1}}{h_{1}}+\cdots+\frac{k_{r} m_{r}}{h_{r}}\right) \cdot Z\left(A_{1}^{k_{1}} \cdots A_{r}^{k_{r}}, s\right) .
\end{aligned}
$$

Thus applying (5.5) we obtain

$$
\begin{aligned}
w(d) & \left(\frac{\sqrt{-d}}{2 \pi}\right)^{s} \Gamma(s) L(M, s) \\
= & \sum_{\substack{0 \leq k_{1}<h_{1} \\
\cdots \\
0 \leq k_{r}<h_{r}}} \cos 2 \pi\left(\frac{k_{1} m_{1}}{h_{1}}+\cdots+\frac{k_{r} m_{r}}{h_{r}}\right) \cdot\left(\frac{\sqrt{-d}}{2 \pi}\right)^{s} \Gamma(s) Z\left(A_{1}^{k_{1}} \cdots A_{r}^{k_{r}}, s\right) \\
= & \sum_{\substack{0 \leq k_{1}<h_{1} \\
0 \leq k_{r}<h_{r}}} \cos 2 \pi\left(\frac{k_{1} m_{1}}{h_{1}}+\cdots+\frac{k_{r} m_{r}}{h_{r}}\right) \cdot\left(\frac{\sqrt{-d}}{2 \pi}\right)^{1-s} \\
& \times \Gamma(1-s) Z\left(A_{1}^{k_{1}} \cdots A_{r}^{k_{r}}, 1-s\right) \\
= & w(d)\left(\frac{\sqrt{-d}}{2 \pi}\right)^{1-s} \Gamma(1-s) L(M, 1-s) .
\end{aligned}
$$

So (ii) is true and the proof is complete. 
Let $d$ be a discriminant with conductor $f$, and let $H(d)$ be given by (5.1). For $K \in H(d)$ we use $R(K)$ to denote the set of integers represented by forms in $K$. Let $p$ be a prime not dividing the conductor $f$. When $p \mid d$, from [MW, Lemma 5.3] (or [SW, Theorem 8.1(ii)]) we know that $p$ is represented by exactly one class $A \in H(d)$ and $A=A_{1}^{\varepsilon_{1} h_{1} / 2} \cdots A_{r}^{\varepsilon_{r} h_{r} / 2}$ with $\varepsilon_{1}, \ldots, \varepsilon_{r} \in$ $\{0,1\}$.

Let $M=A_{1}^{m_{1}} \cdots A_{r}^{m_{r}} \in H(d)$ and $t \in \mathbb{N} \cup\{0\}$. By [SW, Theorem 8.1] we have

$$
\begin{aligned}
& F\left(M, p^{t}\right) \\
& = \begin{cases}\left(1+(-1)^{t}\right) / 2 & \text { if }\left(\frac{d}{p}\right)=-1, \\
(-1)^{t \sum_{j=1}^{r} \varepsilon_{j} m_{j}} & \text { if } p \mid d \text { and } p \in R\left(A_{1}^{\varepsilon_{1} h_{1} / 2} \cdots A_{r}^{\varepsilon_{r} h_{r} / 2}\right), \\
U_{t}\left(\cos 2 \pi \sum_{j=1}^{r} \frac{a_{j} m_{j}}{h_{j}}\right) & \text { if } p \nmid d \text { and } p \in R\left(A_{1}^{a_{1}} \cdots A_{r}^{a_{r}}\right),\end{cases}
\end{aligned}
$$

where $\left\{U_{n}(x)\right\}$ is the Chebyshev polynomial of the second kind given by

$$
U_{0}(x)=1, \quad U_{1}(x)=2 x, \quad U_{n+1}(x)=2 x U_{n}(x)-U_{n-1}(x) \quad(n \geq 1) .
$$

It is well known that (cf. [MOS, p. 259])

$$
\sum_{n=0}^{\infty} U_{n}(x) q^{n}=\frac{1}{1-2 x q+q^{2}} \quad(|x|<1,|q|<1) .
$$

Thus, if $p$ is a prime such that $\left(\frac{d}{p}\right)=1$ and so $p$ is represented by some class $A_{1}^{a_{1}} \cdots A_{r}^{a_{r}} \in H(d)$, then for $s \in \mathbb{C}$ with $\operatorname{Re}(s)>1$ we have

$$
\begin{aligned}
1+\sum_{t=1}^{\infty} F\left(A_{1}^{m_{1}}\right. & \left.\cdots A_{r}^{m_{r}}, p^{t}\right) p^{-s t} \\
& =\sum_{t=0}^{\infty} U_{t}\left(\cos 2 \pi\left(\frac{a_{1} m_{1}}{h_{1}}+\cdots+\frac{a_{r} m_{r}}{h_{r}}\right)\right) \cdot p^{-s t} \\
& =\frac{1}{1-2 \cos 2 \pi\left(\frac{a_{1} m_{1}}{h_{1}}+\cdots+\frac{a_{r} m_{r}}{h_{r}}\right) \cdot p^{-s}+p^{-2 s}} .
\end{aligned}
$$

Now we are in a position to give

THEOREM 5.2. Let $d$ be a discriminant with conductor $f$. Let $H(d)$ be given by (5.1). Let $M=A_{1}^{m_{1}} \cdots A_{r}^{m_{r}} \in H(d)$ and $s \in \mathbb{C}$ with $\operatorname{Re}(s)>1$. Then 


$$
\begin{aligned}
& \sum_{\substack{n=1 \\
(n, f)=1}}^{\infty} \frac{F(M, n)}{n^{s}} \\
& =\prod_{\left(\frac{d}{p}\right)=-1} \frac{1}{1-p^{-2 s}} \prod_{\substack{p \in R\left(A_{1}^{\varepsilon_{1} h_{1} / 2} \cdots A_{r}^{\varepsilon_{r} h_{r} / 2}\right) \\
p \mid d, p \nmid f}} \frac{1}{1-(-1)^{\varepsilon_{1} m_{1}+\cdots+\varepsilon_{r} m_{r}} p^{-s}}
\end{aligned}
$$

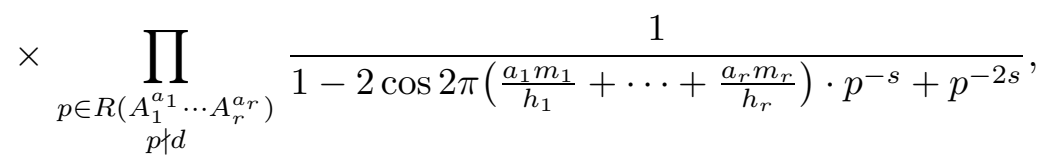

where $\varepsilon_{1}, \ldots, \varepsilon_{r} \in\{0,1\}$ are chosen such that $p \in R\left(A_{1}^{\varepsilon_{1} h_{1} / 2} \cdots A_{r}^{\varepsilon_{r} h_{r} / 2}\right)$ when $p \mid d$ and $p \nmid f$, and $a_{1}, \ldots, a_{r} \in \mathbb{Z}$ are chosen so that $p \in R\left(A_{1}^{a_{1}} \ldots\right.$ $\cdots A_{r}^{a_{r}}$ ) when $\left(\frac{d}{p}\right)=1$.

Proof. Since $F(M, n)$ is a multiplicative function of $n$ (see [SW, Theorem $7.2]$ ), by the argument similar to the proof of Theorem 5.1(i) we see that

$$
\prod_{p \nmid f}\left(1+\sum_{t=1}^{\infty} F\left(M, p^{t}\right) p^{-s t}\right)=\sum_{\substack{n=1 \\(n, f)=1}}^{\infty} \frac{F(M, n)}{n^{s}} .
$$

Let $p$ be a prime not dividing $f$. If $\left(\frac{d}{p}\right)=-1$, it follows from (5.6) that

$$
1+\sum_{t=1}^{\infty} F\left(M, p^{t}\right) p^{-s t}=\sum_{t=0}^{\infty} \frac{1+(-1)^{t}}{2} p^{-s t}=\frac{1}{1-p^{-2 s}} .
$$

If $p \mid d$, then $p$ is represented by exactly one class $A$ in $H(d)$, and $A=$ $A_{1}^{\varepsilon_{1} h_{1} / 2} \ldots A_{r}^{\varepsilon_{r} h_{r} / 2}$ with $\varepsilon_{1}, \ldots, \varepsilon_{r} \in\{0,1\}$. By (5.6) we obtain

$$
1+\sum_{t=1}^{\infty} F\left(M, p^{t}\right) p^{-s t}=\sum_{t=0}^{\infty}(-1)^{t \sum_{j=1}^{r} \varepsilon_{j} m_{j}} p^{-s t}=\frac{1}{1-(-1)^{\sum_{j=1}^{r} \varepsilon_{j} m_{j}} p^{-s}} .
$$

If $\left(\frac{d}{p}\right)=1$ so that $p$ is represented by some class $A_{1}^{a_{1}} \cdots A_{r}^{a_{r}} \in H(d)$, by (5.9) we have

$$
1+\sum_{t=1}^{\infty} F\left(M, p^{t}\right) p^{-s t}=\frac{1}{1-2 \cos 2 \pi\left(\frac{a_{1} m_{1}}{h_{1}}+\cdots+\frac{a_{r} m_{r}}{h_{r}}\right) \cdot p^{-s}+p^{-2 s}} .
$$

Now putting all the above together we deduce the desired result.

From Theorem 5.2 we have

COROLlary 5.1. Let $d$ be a discriminant with conductor $f$ and $2 \nmid h(d)$. Let $H(d)$ be given by (5.1). For $M=A_{1}^{m_{1}} \cdots A_{r}^{m_{r}} \in H(d)$ and $s \in \mathbb{C}$ with $\operatorname{Re}(s)>1$ we have 


$$
\begin{aligned}
& \sum_{\substack{n=1 \\
(n, f)=1}}^{\infty} \frac{F(M, n)}{n^{s}} \\
& \quad=\prod_{\substack{\left(\frac{d}{p}\right)=-1 \\
1-p^{-2 s}}} \frac{1}{p \mid d, p \nmid f} \frac{1}{1-p^{-s}} \\
& \quad \times \prod_{p \in R\left(A_{1}^{\left.a_{1} \ldots A_{r}^{a_{r}}\right)}\right.} \frac{1}{1-2 \cos 2 \pi\left(\frac{a_{1} m_{1}}{h_{1}}+\cdots+\frac{a_{r} m_{r}}{h_{r}}\right) \cdot p^{-s}+p^{-2 s}},
\end{aligned}
$$

where $a_{1}, \ldots, a_{r} \in \mathbb{Z}$ are determined by $p \in R\left(A_{1}^{a_{1}} \cdots A_{r}^{a_{r}}\right)$ when $\left(\frac{d}{p}\right)=1$.

Proof. If $p$ is a prime such that $p \mid d$ and $p \nmid f$, then we must have $p \in R(I)$ since $2 \nmid h(d)$. Now the result follows from Theorem 5.2.

Let $d$ be a discriminant with conductor $f$. Suppose $m \in \mathbb{N}$ and $m \mid f$. In [SW, Lemma 2.1] we showed that any class in $H(d)$ can be written as $\left[a, b m, c m^{2}\right]$, where $a, b, c \in \mathbb{Z},(a, m)=1$ and $\operatorname{gcd}(a, b, c)=1$. Following $[\mathrm{SW}]$ and $[\mathrm{KW} 1]$ we define $\varphi_{1, m}\left(\left[a, b m, \mathrm{~cm}^{2}\right]\right)=[a, b, c]$. From $[\mathrm{SW}$, Theorem 2.1] or [KW1, p. 355] we know that $\varphi_{1, m}$ is a surjective homomorphism from $H(d)$ to $H\left(d / m^{2}\right)$. Thus, if $H(d)$ is cyclic with generator $A$, then $\varphi_{1, m}(A)$ is a generator of $H\left(d / m^{2}\right)$.

THEOREM 5.3. Let $d$ be a discriminant with conductor $f$ and $d_{0}=d / f^{2}$. Suppose that $H(d)$ is cyclic with generator $A$ and order $h$. Let $k \in \mathbb{Z}$ and $s \in \mathbb{C}$ with $\operatorname{Re}(s)>1$. For a prime p let $\alpha_{p}=\operatorname{ord}_{p} f, h_{p}=h\left(d / p^{2 \alpha_{p}}\right)$, and let $\beta_{k, p}$ denote the maximum $j \in\left\{0,1, \ldots, \alpha_{p}\right\}$ such that $h / h\left(d / p^{2 j}\right) \mid k$. Then

$$
\begin{aligned}
& L\left(A^{k}, s\right) \\
& =\prod_{p \nmid f} \frac{1}{1-\left(1+\left(\frac{d_{0}}{p}\right)\right) \cos \frac{2 \pi k a_{p}}{h} p^{-s}+\left(\frac{d_{0}}{p}\right) p^{-2 s}} \prod_{\substack{p \mid f \\
h \nmid k h_{p}}} \frac{1-p^{(1-2 s)\left(1+\beta_{k, p}\right)}}{1-p^{1-2 s}} \\
& \quad \times \prod_{\substack{p|f \\
h| k h_{p}}}\left(\frac{1-p^{\alpha_{p}(1-2 s)}}{1-p^{1-2 s}}+\frac{p^{\alpha_{p}(1-2 s)-1}\left(p-\left(\frac{d_{0}}{p}\right)\right)}{1-\left(1+\left(\frac{d_{0}}{p}\right)\right) \cos \frac{2 \pi k a_{p}}{h} p^{-s}+\left(\frac{d_{0}}{p}\right) p^{-2 s}}\right),
\end{aligned}
$$

where $a_{p} \in \mathbb{Z}$ is uniquely determined by $0 \leq a_{p} \leq h_{p} / 2, p \in R\left(A_{p}^{a_{p}}\right)$ and $A_{p}=\varphi_{1, p^{\alpha}}(A)$.

Proof. Let $p$ be a prime such that $p \mid f\left(\right.$ that is $\alpha_{p} \in \mathbb{N}$ ). Since

$$
h\left(d / p^{2 \beta_{k, p}}\right) \mid h\left(d / p^{2 j}\right) \quad \text { for } j \leq \beta_{k, p},
$$


we see that

$$
h / h\left(d / p^{2 j}\right) \mid k \quad \text { for } j=0,1, \ldots, \beta_{k, p} .
$$

Thus, applying [SW, Theorem 8.4] we obtain

$$
\begin{aligned}
1+\sum_{t=1}^{2 \alpha_{p}-1} F\left(A^{k}, p^{t}\right) p^{-s t} & \\
& =1+\sum_{j=1}^{\alpha_{p}-1} F\left(A^{k}, p^{2 j}\right) p^{-2 j s}=\sum_{j=0}^{\min \left\{\beta_{k, p}, \alpha_{p}-1\right\}} p^{j} \cdot p^{-2 j s} \\
& =\sum_{j=0}^{\min \left\{\beta_{k, p}, \alpha_{p}-1\right\}} p^{j(1-2 s)}=\frac{1-p^{\min \left\{\beta_{k, p}+1, \alpha_{p}\right\}(1-2 s)}}{1-p^{1-2 s}} .
\end{aligned}
$$

Now suppose $t \in \mathbb{N}$ and $t \geq 2 \alpha_{p}$. From [SW, Theorem 8.3(ii)] (with $n=p^{t}$, $m=p^{\alpha_{p}}$ and $s=k$ ) we know that

$$
F\left(A^{k}, p^{t}\right)= \begin{cases}p^{\alpha_{p}}\left(1-\frac{1}{p}\left(\frac{d_{0}}{p}\right)\right) F\left(A_{p}^{k_{p}}, p^{t-2 \alpha_{p}}\right) & \text { if } \beta_{k, p}=\alpha_{p} \\ 0 & \text { if } \beta_{k, p}<\alpha_{p}\end{cases}
$$

where

$$
A_{p}=\varphi_{1, p^{\alpha_{p}}}(A) \in H\left(d / p^{2 \alpha_{p}}\right) \quad \text { and } \quad k_{p}=k h_{p} / h \in \mathbb{Z}
$$

Thus, if $\beta_{k, p}=\alpha_{p}$, then

$$
\begin{aligned}
\sum_{t=2 \alpha_{p}}^{\infty} F\left(A^{k}, p^{t}\right) p^{-s t} & =p^{\alpha_{p}}\left(1-\frac{1}{p}\left(\frac{d_{0}}{p}\right)\right) \sum_{t=2 \alpha_{p}}^{\infty} F\left(A_{p}^{k_{p}}, p^{t-2 \alpha_{p}}\right) p^{-s t} \\
& =p^{\alpha_{p}(1-2 s)}\left(1-\frac{1}{p}\left(\frac{d_{0}}{p}\right)\right) \sum_{j=0}^{\infty} F\left(A_{p}^{k_{p}}, p^{j}\right) p^{-j s} .
\end{aligned}
$$

Since $\varphi_{1, p^{\alpha} \alpha_{p}}$ is a surjective homomorphism from $H(d)$ to $H\left(d / p^{2 \alpha_{p}}\right)$, we see that $A_{p}$ is a generator of $H\left(d / p^{2 \alpha_{p}}\right)$. Note that

$$
d / p^{2 \alpha_{p}}=d_{0}\left(f / p^{\alpha_{p}}\right)^{2}, \quad p \nmid f / p^{\alpha_{p}} \quad \text { and } \quad k / h=k_{p} / h_{p} .
$$

By the proof of Theorem 5.2 the sum

$$
1+\sum_{j=1}^{\infty} F\left(A_{p}^{k_{p}}, p^{j}\right) p^{-j s}
$$


is equal to

$$
\begin{cases}\frac{1}{1-p^{-2 s}} & \text { if }\left(\frac{d_{0}}{p}\right)=-1 \\ \frac{1}{1-(-1)^{\varepsilon_{p} k_{p} p^{-s}}} & \text { if } p \mid d_{0} \text { and } p \in R\left(A_{p}^{\varepsilon_{p} h_{p} / 2}\right), \\ \frac{1}{1-2 \cos \frac{2 \pi a_{p} k_{p}}{h_{p}} p^{-s}+p^{-2 s}} & \text { if }\left(\frac{d_{0}}{p}\right)=1 \text { and } p \in R\left(A_{p}^{a_{p}}\right), \\ =\frac{1}{1-\left(1+\left(\frac{d_{0}}{p}\right)\right) \cos \frac{2 \pi k a_{p}}{h} p^{-s}+\left(\frac{d_{0}}{p}\right) p^{-2 s}},\end{cases}
$$

where $\varepsilon_{p} \in\{0,1\}$ is given by $p \in R\left(A_{p}^{\varepsilon_{p} h_{p} / 2}\right)$ when $p \mid d_{0}$, and $a_{p} \in \mathbb{Z}$ is determined by $0 \leq a_{p} \leq h_{p} / 2$ and $p \in R\left(A_{p}^{a_{p}}\right)$ when $\left(\frac{d_{0}}{p}\right)=0,1$. Thus

$$
\begin{aligned}
& 1+\sum_{t=1}^{\infty} F\left(A^{k}, p^{t}\right) p^{-s t}=1+\sum_{t=1}^{2 \alpha_{p}-1} F\left(A^{k}, p^{t}\right) p^{-s t}+\sum_{t=2 \alpha_{p}}^{\infty} F\left(A^{k}, p^{t}\right) p^{-s t} \\
& = \begin{cases}\frac{1-p^{(1-2 s)\left(1+\beta_{k, p}\right)}}{1-p^{1-2 s}} & \text { if } \beta_{k, p}<\alpha_{p}, \\
\frac{1-p^{\alpha_{p}(1-2 s)}}{1-p^{1-2 s}}+\frac{p^{\alpha_{p}(1-2 s)-1}\left(p-\left(\frac{d_{0}}{p}\right)\right)}{1-\left(1+\left(\frac{d_{0}}{p}\right)\right) \cos \frac{2 \pi k a_{p}}{h} p^{-s}+\left(\frac{d_{0}}{p}\right) p^{-2 s}} & \text { if } \beta_{k, p}=\alpha_{p} .\end{cases}
\end{aligned}
$$

By (5.10) and Theorem 5.2 we have

$$
\begin{aligned}
\prod_{p \nmid f}\left(1+\sum_{t=1}^{\infty} F\left(A^{k}, p^{t}\right) p^{-s t}\right)= & \prod_{\substack{\left(\frac{d}{p}\right)=-1 \\
1-p^{-2 s}}} \frac{1}{\substack{p \in R\left(A^{\varepsilon h / 2}\right) \\
p \mid d, p \nmid f}} \frac{1}{1-(-1)^{k \varepsilon} p^{-s}} \\
& \times \prod_{\substack{p \in R\left(A^{a}\right) \\
\left(\frac{d}{p}\right)=1}} \frac{1}{1-2 \cos \frac{2 \pi k a}{h} p^{-s}+p^{-2 s}} \\
= & \prod_{p \nmid f} \frac{1}{1-\left(1+\left(\frac{d_{0}}{p}\right)\right) \cos \frac{2 \pi k a_{p}}{h} p^{-s}+\left(\frac{d_{0}}{p}\right) p^{-2 s}},
\end{aligned}
$$

where $\varepsilon \in\{0,1\}$ is given by $p \in R\left(A^{\varepsilon h / 2}\right)$ when $p \mid d$, and $a \in \mathbb{Z}$ is determined by $0 \leq a \leq h / 2$ and $p \in R\left(A^{a}\right)$ when $\left(\frac{d}{p}\right)=0,1$.

Note that $A_{p}=A$ when $p \nmid f$. Putting all the above together with Theorem 5.1(i) gives the result.

Corollary 5.2. Let $d$ be a discriminant with conductor $f$ and $d_{0}=$ $d / f^{2}$. Suppose that $H(d)$ is cyclic with generator $A$ and $h=h(d) \equiv 1$ $(\bmod 2)$. Let $k \in \mathbb{Z}$. For a prime $p$ let $\alpha_{p}=\operatorname{ord}_{p} f, h_{p}=h\left(d / p^{2 \alpha_{p}}\right)$, and let $\beta_{k, p}$ denote the maximum number $j \in\left\{0,1, \ldots, \alpha_{p}\right\}$ such that $h / h\left(d / p^{2 j}\right) \mid k$. 
For $s \in \mathbb{C}$ with $\operatorname{Re}(s)>1$ we have

$$
\begin{aligned}
& L\left(A^{k}, s\right) \\
& =\prod_{\left(\frac{d}{p}\right)=-1} \frac{1}{1-p^{-2 s}} \prod_{p \mid d, p \nmid f} \frac{1}{1-p^{-s}} \prod_{\substack{p \in R\left(A^{a}\right) \\
\left(\frac{d}{p}\right)=1}} \frac{1}{1-2 \cos \frac{2 \pi k a}{h} p^{-s}+p^{-2 s}} \\
& \times \prod_{\substack{p \mid f \\
h \nmid k h_{p}}} \frac{1-p^{(1-2 s)\left(1+\beta_{k, p}\right)}}{1-p^{1-2 s}} \prod_{\substack{p|f, h| k h_{p} \\
\left(\frac{d_{0}}{p}\right)=-1}}\left(\frac{1-p^{\alpha_{p}(1-2 s)}}{1-p^{1-2 s}}+\frac{p^{\alpha_{p}(1-2 s)-1}(p+1)}{1-p^{-2 s}}\right) \\
& \times \prod_{\substack{p|f, h| k h_{p} \\
p \mid d_{0}}}\left(\frac{1-p^{\alpha_{p}(1-2 s)}}{1-p^{1-2 s}}+\frac{p^{\alpha_{p}(1-2 s)}}{1-p^{-s}}\right) \\
& \times \prod_{\substack{p|f, h| k h_{p} \\
\left(\frac{d_{0}}{p}\right)=1, p \in R\left(A_{p}^{a_{p}}\right)}}\left(\frac{1-p^{\alpha_{p}(1-2 s)}}{1-p^{1-2 s}}+\frac{p^{\alpha_{p}(1-2 s)-1}(p-1)}{1-2 \cos \frac{2 \pi k a_{p}}{h} p^{-s}+p^{-2 s}}\right),
\end{aligned}
$$

where $a \in \mathbb{Z}$ is determined by $0 \leq a \leq(h-1) / 2$ and $p \in R\left(A^{a}\right)$ when $\left(\frac{d}{p}\right)=1, A_{p}=\varphi_{1, p^{\alpha}}(A)$, and $a_{p} \in \mathbb{Z}$ is determined by $0 \leq a_{p} \leq\left(h_{p}-1\right) / 2$ and $p \in R\left(A_{p}^{a_{p}}\right)$ when $p \mid f$ and $\left(\frac{d_{0}}{p}\right)=1$.

Proof. If $p$ is a prime such that $p \mid d$ and $p \nmid f$, from [MW, Lemma 5.3] or [SW, Lemma 5.2] we know that $p$ is represented by unique class $M$ in $H(d)$ and $M=M^{-1}$. Thus we must have $p \in R(I)$ since $H(d)$ is cyclic and $2 \nmid h(d)$. Now the result follows from Theorem 5.3.

THEOREM 5.4. Let $d$ be a discriminant such that $h(d)=5$. Let $f$ be the conductor of $d$, and let $A$ be a generator of $H(d)$. Let

$$
\begin{gathered}
F(A, n)=\frac{1}{w(d)}\left(R(I, n)+\frac{\sqrt{5}-1}{2} R(A, n)-\frac{\sqrt{5}+1}{2} R\left(A^{2}, n\right)\right), \\
F\left(A^{2}, n\right)=\frac{1}{w(d)}\left(R(I, n)-\frac{\sqrt{5}+1}{2} R(A, n)+\frac{\sqrt{5}-1}{2} R\left(A^{2}, n\right)\right) .
\end{gathered}
$$

For $s \in \mathbb{C}$ with $\operatorname{Re}(s)>1$ we have

$$
\begin{aligned}
\sum_{\substack{n=1 \\
(n, f)=1}}^{\infty} \frac{F(A, n)}{n^{s}}= & \prod_{\substack{\left.\frac{d}{p}\right)=-1 \\
1-p^{-2 s}}} \frac{1}{\begin{array}{c}
p \mid d \\
p \nmid f
\end{array}} \frac{1}{1-p^{-s}} \prod_{\substack{p \in R(I) \\
p \nmid d}} \frac{1}{\left(1-p^{-s}\right)^{2}} \\
& \times \prod_{p \in R(A)} \frac{1}{1-\frac{\sqrt{5}-1}{2} p^{-s}+p^{-2 s}} \prod_{p \in R\left(A^{2}\right)} \frac{1}{1+\frac{\sqrt{5}+1}{2} p^{-s}+p^{-2 s}}
\end{aligned}
$$




$$
\begin{aligned}
\sum_{\substack{n=1 \\
(n, f)=1}}^{\infty} \frac{F\left(A^{2}, n\right)}{n^{s}}= & \prod_{\substack{\left(\frac{d}{p}\right)=-1 \\
1-p^{-2 s}}} \frac{1}{\substack{p \mid d \\
p \nmid f}} \frac{1}{1-p^{-s}} \prod_{\substack{p \in R(I) \\
p \nmid d}} \frac{1}{\left(1-p^{-s}\right)^{2}} \\
& \times \prod_{p \in R\left(A^{2}\right)} \frac{1}{1-\frac{\sqrt{5}-1}{2} p^{-s}+p^{-2 s}} \prod_{p \in R(A)} \frac{1}{1+\frac{\sqrt{5}+1}{2} p^{-s}+p^{-2 s}}
\end{aligned}
$$

Proof. The result follows from Corollary 5.1, [SW, Theorem 7.4] and the facts

$$
\cos \frac{2 \pi}{5}=\sin \frac{\pi}{10}=\frac{\sqrt{5}-1}{4}, \quad \cos \frac{4 \pi}{5}=-\cos \frac{\pi}{5}=-\frac{\sqrt{5}+1}{4} .
$$

From Theorem 5.2 and [SW, Theorem 7.4] we can easily deduce

THEOREM 5.5. Let $d$ be a discriminant such that $h(d)=6$. Let $f$ be the conductor of $d$, and let $A$ be a generator of $H(d)$. Let

$$
\begin{gathered}
F(A, n)=\frac{1}{w(d)}\left(R(I, n)+R(A, n)-R\left(A^{2}, n\right)-R\left(A^{3}, n\right)\right), \\
F\left(A^{2}, n\right)=\frac{1}{w(d)}\left(R(I, n)-R(A, n)-R\left(A^{2}, n\right)+R\left(A^{3}, n\right)\right), \\
F\left(A^{3}, n\right)=\frac{1}{w(d)}\left(R(I, n)-2 R(A, n)+2 R\left(A^{2}, n\right)-R\left(A^{3}, n\right)\right) .
\end{gathered}
$$

For $j=1,2,3$ and $s \in \mathbb{C}$ with $\operatorname{Re}(s)>1$ we have

$$
\begin{aligned}
\sum_{\substack{n=1 \\
(n, f)=1}}^{\infty} & \frac{F\left(A^{j}, n\right)}{n^{s}} \\
= & \prod_{\substack{\left(\frac{d}{p}\right)=-1 \\
1-p^{-2 s}}} \frac{1}{\substack{p \in R(I) \\
p \mid d, p \nmid f}} \frac{1}{1-p^{-s}} \prod_{\substack{p \in R\left(A^{3}\right) \\
p \mid d, p \nmid f}} \frac{1}{1-(-1)^{j} p^{-s}} \\
& \times \prod_{\substack{p \in R(I) \\
p \nmid d}} \frac{1}{\left(1-p^{-s}\right)^{2}} \prod_{p \in R(A)} \frac{1}{1-c_{j} p^{-s}+p^{-2 s}} \\
& \times \prod_{p \in R\left(A^{2}\right)} \frac{1}{1-(-1)^{j} c_{j} p^{-s}+p^{-2 s}} \prod_{\substack{p \in R\left(A^{3}\right) \\
p \nmid d}} \frac{1}{\left(1-(-1)^{j} p^{-s}\right)^{2}},
\end{aligned}
$$

where

$$
c_{j}=2 \cos \frac{j \pi}{3}= \begin{cases}1 & \text { if } j=1 \\ -1 & \text { if } j=2, \\ -2 & \text { if } j=3\end{cases}
$$


6. The Euler product for $L(A, s)$ when $H(d)=\{I, A\}$. Let $d$ be a discriminant with $h(d)=2$. Suppose $I$ is the principal class and $A$ is the generator of $H(d)$. We recall that

and

$$
F(A, n)= \begin{cases}R(I, n)-R(A, n) & \text { if } d>0 \\ \frac{1}{2}(R(I, n)-R(A, n)) & \text { if } d<0\end{cases}
$$

$$
L(A, s)=\sum_{n=1}^{\infty} \frac{F(A, n)}{n^{s}} \quad(\operatorname{Re}(s)>1) .
$$

Putting $h=2$ and $k=1$ in Theorem 5.3 we deduce

THEOREM 6.1. Let $d$ be a discriminant with conductor $f$. Suppose $h(d)$ $=2$ and $H(d)=\{I, A\}$. For a prime $p$ let $\alpha_{p}$ be the nonnegative integer such that $p^{\alpha_{p}} \| f, h_{p}=h\left(d / p^{2 \alpha_{p}}\right)$, and let $\beta_{p}$ denote the maximum $j \in$ $\left\{0,1, \ldots, \alpha_{p}\right\}$ such that $h\left(d / p^{2 j}\right)=2$. Then for $s \in \mathbb{C}$ with $\operatorname{Re}(s)>1$ we have

$$
\begin{aligned}
L(A, s)= & \prod_{\substack{p \mid d, p \nmid f \\
p \in R(I)}} \frac{1}{1-p^{-s}} \prod_{\substack{p \mid d, p \nmid f \\
p \in R(A)}} \frac{1}{1+p^{-s}} \prod_{\substack{\left.\frac{d}{p}\right)=-1 \\
1-p^{-2 s}}} \frac{1}{1} \\
& \times \prod_{\substack{p \in R(I) \\
p \nmid d}} \frac{1}{\left(1-p^{-s}\right)^{2}} \prod_{\substack{p \in R(A) \\
p \nmid d}} \frac{1}{\left(1+p^{-s}\right)^{2}} \prod_{\substack{p \mid f \\
h_{p}=1}} \frac{1-p^{(1-2 s)\left(1+\beta_{p}\right)}}{1-p^{1-2 s}} \\
& \times \prod_{\substack{p \mid f \\
h_{p}=2}}\left(\frac{1-p^{\alpha_{p}(1-2 s)}}{1-p^{1-2 s}}+\frac{p^{\alpha_{p}(1-2 s)-1}\left(p-\left(\frac{d_{0}}{p}\right)\right)}{1-(-1)^{a_{p}}\left(1+\left(\frac{d_{0}}{p}\right)\right) p^{-s}+\left(\frac{d_{0}}{p}\right) p^{-2 s}}\right),
\end{aligned}
$$

where $a_{p}=0$ or 1 according as $p$ is represented by the principal class in $H\left(d / p^{2 \alpha_{p}}\right)$ or not.

TheOREM 6.2. Let $d<0$ be a discriminant with $h(d)=2$, which is given in [SW, Table 9.1]. Let $f$ be the conductor of $d$ and $H(d)=\{I, A\}$ with $A^{2}=I$. Let $s \in \mathbb{C}$ with $\operatorname{Re}(s)>1$.

(i) If $d \neq-60$, then

$$
\begin{aligned}
L(A, s)= & \prod_{\substack{\left.\frac{d}{p}\right)=-1\\
}} \frac{1}{1-p^{-2 s}} \prod_{\substack{p \mid d, p \nmid f \\
p \in R(I)}} \frac{1}{1-p^{-s}} \prod_{\substack{p \mid d, p \nmid f \\
p \in R(A)}} \frac{1}{1+p^{-s}} \\
& \times \prod_{\substack{p \in R(I) \\
p \nmid d}} \frac{1}{\left(1-p^{-s}\right)^{2}} \prod_{\substack{p \in R(A) \\
p \nmid d}} \frac{1}{\left(1+p^{-s}\right)^{2}},
\end{aligned}
$$

where $I, A$ and the conditions for $p \in R(I)$ and $p \in R(A)$ are given in [SW, Table 9.1]. 
(ii) If $d=-60$, then $I=[1,0,15], A=[3,0,5]$ and

$$
\begin{aligned}
& L(A, s) \\
& =\frac{1+2^{1-s}+2^{1-2 s}}{\left(1+2^{-s}\right)^{2}} \cdot \frac{1}{1+3^{-s}} \cdot \frac{1}{1+5^{-s}} \prod_{p \equiv 7,11,13,14(\bmod 15)} \frac{1}{1-p^{-2 s}} \\
& \quad \times \prod_{p \equiv 1,4(\bmod 15)} \frac{1}{\left(1-p^{-s}\right)^{2}} \prod_{\substack{p \equiv 2,8(\bmod 15) \\
p \neq 2}} \frac{1}{\left(1+p^{-s}\right)^{2}} .
\end{aligned}
$$

Proof. If $d \neq-60$ and $p$ is a prime such that $p \mid f$, by [SW, Tables 9.1, 9.2 and (9.3)] we see that $h\left(d / p^{2}\right)=1$. Hence $h_{p}=h\left(d / p^{2 \alpha_{p}}\right)=1$ for $\alpha_{p}=\operatorname{ord}_{p} f$ by [SW, Theorem 8.3(ii)]. Now applying Theorem 6.1 and [SW, Table 9.1] we obtain (i).

Now suppose $d=-60$. Then $f=2$ and $d_{0}=d / f^{2}=-15$. Let $p$ be an odd prime. By [SW, Table 9.1] we have $H(-60)=\{[1,0,15],[3,0,5]\}$, $H(-15)=\{[1,1,4],[2,1,2]\}, p \in R([1,0,15]) \Leftrightarrow p \equiv 1,4(\bmod 15) \Leftrightarrow p \in$ $R([1,1,4])$, and $p \in R([3,0,5]) \Leftrightarrow p \in R([2,1,2]) \Leftrightarrow p=3,5$ or $p \equiv 2,8$ $(\bmod 15)$. If $p$ is a prime such that $p \mid f$, we must have $p=2, \alpha_{p}=1$, $h_{p}=2$ and $\left(\frac{d_{0}}{p}\right)=\left(\frac{-15}{2}\right)=1$. Thus applying Theorem 6.1 and the above we obtain (ii). The proof is now complete.

Theorem 6.3. Let $s \in \mathbb{C}$ with $\operatorname{Re}(s)>1$. Then

$$
\begin{aligned}
\sum_{n=1}^{\infty} \frac{\psi_{2}(n)}{n^{s}}= & \frac{1}{1+3^{-s}} \prod_{p \equiv 5(\bmod 6)} \frac{1}{1-p^{-2 s}} \prod_{p \equiv 1(\bmod 12)} \frac{1}{\left(1-p^{-s}\right)^{2}} \\
& \times \prod_{p \equiv 7(\bmod 12)} \frac{1}{\left(1+p^{-s}\right)^{2}}
\end{aligned}
$$

and

$$
\begin{aligned}
\sum_{n=1}^{\infty} \frac{\psi_{4}(n)}{n^{s}} & \\
& =\prod_{p \equiv 3(\bmod 4)} \frac{1}{1-p^{-2 s}} \prod_{p \equiv 1(\bmod 8)} \frac{1}{\left(1-p^{-s}\right)^{2}} \prod_{p \equiv 5(\bmod 8)} \frac{1}{\left(1+p^{-s}\right)^{2}} .
\end{aligned}
$$

Proof. From [SW, Table 9.1] we see that $H(-48)=\{[1,0,12],[3,0,4]\}$ and $H(-64)=\{[1,0,16],[4,4,5]\}$. Thus $\psi_{2}(n)=F([3,0,4], n)$ and $\psi_{4}(n)=$ $F([4,4,5], n)$ by Theorem 2.1. Now applying Theorem 6.2(i) and [SW, Table 9.1] we obtain the result. 
Theorem 6.4. For $n \in \mathbb{N}$ we have

$$
f_{4}(1,4 ; n)+f_{4}(3,4 ; n)= \begin{cases}(-1)^{(n-1) / 2} \sum_{m \mid n}\left(\frac{m}{7}\right) & \text { if } 2 \nmid n, \\ 0 & \text { if } 2 \mid n .\end{cases}
$$

For $s \in \mathbb{C}$ with $\operatorname{Re}(s)>1$ we have

$$
\begin{aligned}
\sum_{n=1}^{\infty} \frac{f_{4}(1,4 ; n)+f_{4}(3,4 ; n)}{n^{s}} & \frac{1}{1+7^{-s}} \prod_{p \equiv 3,5,6(\bmod 7)} \frac{1}{1-p^{-2 s}} \prod_{p \equiv 1,9,25(\bmod 28)} \frac{1}{\left(1-p^{-s}\right)^{2}} \\
& \times \prod_{p \equiv 11,15,23(\bmod 28)} \frac{1}{\left(1+p^{-s}\right)^{2}} .
\end{aligned}
$$

Proof. From Theorem 2.3 and [SW, Table 9.1] we see that

$$
f_{4}(1,4 ; n)+f_{4}(3,4 ; n)=\frac{1}{2}(R(1,0,28 ; n)-R(4,0,7 ; n))=F([4,0,7], n) .
$$

But according to [SW, Theorem 9.2] and Lemma 3.1,

$$
F([4,0,7], n)= \begin{cases}(-1)^{(n-1) / 2} \sum_{m \mid n}\left(\frac{-7}{m}\right)=(-1)^{(n-1) / 2} \sum_{m \mid n}\left(\frac{m}{7}\right) & \text { if } 2 \nmid n, \\ 0 & \text { if } 2 \mid n .\end{cases}
$$

Thus the result follows from Theorem 6.2 and [SW, Table 9.1].

Remark 6.1. Comparing Theorems 4.1 and 6.4 we conclude that $f_{4}(1,4 ; n)+f_{4}(3,4 ; n)=(-1)^{(n-1) / 2} \psi_{1}(n)$ for odd $n$.

7. The Euler product for $L(A, s)$ when $H(d)=\left\{I, A, A^{2}\right\}$. Let $d$ be a discriminant with $h(d)=3$. Suppose $I$ is the principal class and $A$ is a generator of $H(d)$. We recall that

$$
F(A, n)=\frac{1}{w(d)}(R(I, n)-R(A, n))= \begin{cases}R(I, n)-R(A, n) & \text { if } d>0, \\ \frac{1}{2}(R(I, n)-R(A, n)) & \text { if } d<0\end{cases}
$$

and

$$
L(A, s)=\sum_{n=1}^{\infty} \frac{F(A, n)}{n^{s}} \quad(\operatorname{Re}(s)>1) .
$$

THEOREM 7.1. Let $d<0$ be a discriminant with $h(d)=3$. (The values of such d are given for example in [WH, Proposition] or [SW, Lemma 10.1].) Let $f$ be the conductor of $d$ and $H(d)=\left\{I, A, A^{2}\right\}$ with $A^{3}=I$. Let $s \in \mathbb{C}$ with $\operatorname{Re}(s)>1$. 
(i) If $d \neq-92,-124$, then

$$
\begin{aligned}
L(A, s)= & \prod_{\substack{p \mid d \\
p \nmid f}} \frac{1}{1-p^{-s}} \prod_{\left(\frac{d}{p}\right)=-1} \frac{1}{1-p^{-2 s}} \\
& \times \prod_{\substack{p \in R(I) \\
p \nmid d}} \frac{1}{\left(1-p^{-s}\right)^{2}} \prod_{p \in R(A)} \frac{1}{1+p^{-s}+p^{-2 s}} .
\end{aligned}
$$

(ii) If $d=-92$, then $I=[1,0,23], A=[3,2,8]$ and

$$
\begin{aligned}
L(A, s)= & \frac{1+2^{-s}+2^{1-2 s}}{1+2^{-s}+2^{-2 s}} \cdot \frac{1}{1-23^{-s}} \prod_{\left(\frac{p}{23}\right)=-1} \frac{1}{1-p^{-2 s}} \\
& \times \prod_{p=x^{2}+23 y^{2} \neq 23} \frac{1}{\left(1-p^{-s}\right)^{2}} \prod_{p=3 x^{2}+2 x y+8 y^{2}} \frac{1}{1+p^{-s}+p^{-2 s}} .
\end{aligned}
$$

(iii) If $d=-124$, then $I=[1,0,31], A=[5,4,7]$ and

$$
\begin{aligned}
L(A, s)= & \frac{1+2^{-s}+2^{1-2 s}}{1+2^{-s}+2^{-2 s}} \cdot \frac{1}{1-31^{-s}} \prod_{\left(\frac{p}{31}\right)=-1} \frac{1}{1-p^{-2 s}} \\
& \times \prod_{p=x^{2}+31 y^{2} \neq 31} \frac{1}{\left(1-p^{-s}\right)^{2}} \prod_{p=5 x^{2}+4 x y+7 y^{2}} \frac{1}{1+p^{-s}+p^{-2 s}} .
\end{aligned}
$$

Proof. We first suppose $d \neq-92,-124$. Let $p$ be a prime dividing the conductor $f$ and $p^{\alpha_{p}} \| f$. From [SW, (9.3)] and [SW, Lemma 10.1] we know that $h\left(d / p^{2}\right)=1$ and therefore $h\left(d / p^{2 j}\right)=1$ for $j=1, \ldots, \alpha_{p}$ by [SW, Remark 2.2]. Thus putting $h=3$ and $k=1$ in Corollary 5.2 yields the result in this case.

If $d=-92,-124$ and $p$ is a prime such that $p \mid f$, then $p=f=2$, $\left(\frac{d / 4}{p}\right)=1$ and $h\left(d / p^{2}\right)=3$. We note that $H(-92)=\{[1,0,23],[3,2,8]$, $[3,-2,8]\}, H(-124)=\{[1,0,31],[5,4,7],[5,-4,7]\}, H(-23)=\{[1,1,6]$, $[2,1,3],[2,-1,3]\}, H(-31)=\{[1,1,8],[2,1,4],[2,-1,4]\}$. Thus putting $h=3, k=1$ and $d=-92,-124$ in Corollary 5.2 we obtain (ii) and (iii). The proof is now complete.

Theorem 7.2. Let $s \in \mathbb{C}$ with $\operatorname{Re}(s)>1$. Then

$$
\begin{aligned}
\sum_{n=1}^{\infty} \frac{\phi_{1}(n)}{n^{s}}= & \frac{1}{1-23^{-s}} \prod_{\left(\frac{p}{23}\right)=-1} \frac{1}{1-p^{-2 s}} \prod_{p=2 x^{2}+x y+3 y^{2}} \frac{1}{1+p^{-s}+p^{-2 s}} \\
& \times \prod_{p=x^{2}+x y+6 y^{2} \neq 23} \frac{1}{\left(1-p^{-s}\right)^{2}},
\end{aligned}
$$




$$
\begin{aligned}
\sum_{n=1}^{\infty} \frac{\phi_{2}(n)}{n^{s}}= & \frac{1}{1-11^{-s}} \prod_{p \equiv 2,6,7,8,10(\bmod 11)} \frac{1}{1-p^{-2 s}} \\
& \times \prod_{\substack{p=3 x^{2}+2 x y+4 y^{2} \\
1+p^{-s}+p^{-2 s}}} \frac{1}{\left(1-p^{-s}\right)^{2}}
\end{aligned}
$$

and

$$
\begin{aligned}
\sum_{n=1}^{\infty} \frac{\phi_{6}(n)}{n^{s}}= & \prod_{p \equiv 5(\bmod 6)} \frac{1}{1-p^{-2 s}} \prod_{p=x^{2}+27 y^{2}} \frac{1}{\left(1-p^{-s}\right)^{2}} \\
& \times \prod_{p=4 x^{2}+2 x y+7 y^{2}} \frac{1}{1+p^{-s}+p^{-2 s}} .
\end{aligned}
$$

Proof. The result follows from the fact that $h(-23)=h(-44)=h(-108)$ $=3$, (4.1) and Theorem 7.1.

REMARK 7.1. Note that an odd prime $p$ is represented by $x^{2}+x y+6 y^{2}$ if and only if $p$ is represented by $x^{2}+23 y^{2}$. The formula for $\phi_{1}(n)$ in Theorem 7.2 was essentially conjectured by Ramanujan ([R1]). In [Ra], Rangachari outlined a proof of this result using class field theory and modular forms. The formula for $\phi_{2}(n)$ in Theorem 7.2 corrects the incorrect formula of Ramanujan and Rangachari (see $[\mathrm{Ra}]$ ).

TheOrem 7.3. For $s \in \mathbb{C}$ with $\operatorname{Re}(s)>1$ we have

$$
\begin{aligned}
\sum_{n=1}^{\infty} \frac{f_{2}(1,5 ; n)+f_{2}(3,5 ; n)}{n^{s}} & \\
= & \frac{1}{1-19^{-s}} \prod_{\substack{\left(\frac{p}{19}\right)=-1 \\
p \neq 2}} \frac{1}{1-p^{-2 s}} \prod_{p=x^{2}+19 y^{2} \neq 19} \frac{1}{\left(1-p^{-s}\right)^{2}} \\
& \times \prod_{p=4 x^{2}+2 x y+5 y^{2}} \frac{1}{1+p^{-s}+p^{-2 s}} .
\end{aligned}
$$

Proof. From Theorem 2.4 we see that

$$
\begin{aligned}
f_{2}(1,5 ; n)+f_{2}(3,5 ; n) & =\frac{1}{2}(R(1,0,19 ; n)-R(4,2,5 ; n)) \\
& =F([4,2,5], n) .
\end{aligned}
$$

Since $h(-76)=3$, applying Theorem $7.1(\mathrm{i})$ we deduce the result. 
8. Euler products for $L(A, s)$ and $L\left(A^{2}, s\right)$ when $H(d)=\left\{I, A, A^{2}\right.$, $\left.A^{3}\right\}$. Let $d$ be a discriminant such that $H(d)=\left\{I, A, A^{2}, A^{3}\right\}$ with $A^{4}=I$. From [SW, Theorem 7.4] we know that for $n \in \mathbb{N}$,

$$
F(A, n)=\left(R(I, n)-R\left(A^{2}, n\right)\right) / w(d)
$$

and

$$
F\left(A^{2}, n\right)=\left(R(I, n)-2 R(A, n)+R\left(A^{2}, n\right)\right) / w(d) .
$$

Thus for $s \in \mathbb{C}$ with $\operatorname{Re}(s)>1$ we have

$$
L(A, s)=\sum_{n=1}^{\infty} \frac{F(A, n)}{n^{s}}=\sum_{n=1}^{\infty} \frac{\left(R(I, n)-R\left(A^{2}, n\right)\right) / w(d)}{n^{s}}
$$

and

$$
L\left(A^{2}, s\right)=\sum_{n=1}^{\infty} \frac{F\left(A^{2}, n\right)}{n^{s}}=\sum_{n=1}^{\infty} \frac{\left(R(I, n)-2 R(A, n)+R\left(A^{2}, n\right)\right) / w(d)}{n^{s}} .
$$

Let $d$ be a discriminant with conductor $f$. If $p$ is a prime such that $p \mid d$ and $p \nmid f$, then $p$ is represented by a unique class $M$ in $H(d)$ and $M=M^{-1}$. Thus, if $H(d)=\left\{I, A, A^{2}, A^{3}\right\}$ with $A^{4}=I$, then either $p \in R(I)$ or $p \in$ $R\left(A^{2}\right)$.

TheOREM 8.1. Let $d<0$ be a discriminant such that $H(d)=\left\{I, A, A^{2}\right.$, $\left.A^{3}\right\}$ with $A^{4}=I$, which is given in [SW, Proposition 11.1(i)]. Let $f$ be the conductor of $d$ and $s \in \mathbb{C}$ with $\operatorname{Re}(s)>1$.

(i) If $d \neq-220,-252$, then

$$
\begin{aligned}
L(A, s)= & \prod_{\left(\frac{d}{p}\right)=-1} \frac{1}{1-p^{-2 s}} \prod_{\substack{p \mid d, p \nmid f \\
p \in R(I)}} \frac{1}{1-p^{-s}} \prod_{\substack{p \mid d, p \nmid f \\
p \in R\left(A^{2}\right)}} \frac{1}{1+p^{-s}} \\
& \times \prod_{\substack{p \nmid d \\
p \in R(I)}} \frac{1}{\left(1-p^{-s}\right)^{2}} \prod_{\substack{p \nmid d \\
p \in R\left(A^{2}\right)}} \frac{1}{\left(1+p^{-s}\right)^{2}} \prod_{p \in R(A)} \frac{1}{1+p^{-2 s}} .
\end{aligned}
$$

(ii) If $d=-220$ and so $F(A, n)=\frac{1}{2}(R(1,0,55 ; n)-R(5,0,11 ; n))$, then

$$
\begin{aligned}
L(A, s)= & \frac{1+2^{1-2 s}}{1+2^{-2 s}} \cdot \frac{1}{\left(1+5^{-s}\right)\left(1+11^{-s}\right)} \prod_{\left(\frac{p}{11}\right)=-\left(\frac{p}{5}\right)} \frac{1}{1-p^{-2 s}} \\
& \times \prod_{\left(\frac{p}{11}\right)=\left(\frac{p}{5}\right)=-1} \frac{1}{1+p^{-2 s}} \\
& \times \prod_{p=x^{2}+55 y^{2}} \frac{1}{\left(1-p^{-s}\right)^{2}} \prod_{\substack{p=5 x^{2}+11 y^{2} \\
p \neq 5,11}} \frac{1}{\left(1+p^{-s}\right)^{2}} .
\end{aligned}
$$


(iii) If $d=-252$ and so $F(A, n)=\frac{1}{2}(R(1,0,63 ; n)-R(7,0,9 ; n))$, then

$$
\begin{aligned}
L(A, s)= & \frac{1+2^{1-2 s}}{1+2^{-2 s}} \cdot \frac{1}{1+7^{-s}} \prod_{\substack{p \equiv 3,5,6(\bmod 7) \\
p \neq 3}} \frac{1}{1-p^{-2 s}} \\
& \times \prod_{\substack{p \equiv 2,8,11(\bmod 21) \\
p \neq 2}} \frac{1}{1+p^{-2 s}} \\
& \times \prod_{p=x^{2}+63 y^{2}} \frac{1}{\left(1-p^{-s}\right)^{2}} \prod_{\substack{p=7 x^{2}+9 y^{2} \\
p \neq 7}} \frac{1}{\left(1+p^{-s}\right)^{2}} .
\end{aligned}
$$

Proof. Let $p$ be a prime such that $p \mid f$ and $p^{\alpha_{p}} \| f$. If $d \neq-220,-252$, by [SW, Proposition 11.1(i) and Remark 2.2] we have $h\left(d / p^{2}\right)=1,2$ and so $h\left(d / p^{2 \alpha_{p}}\right)=1,2$. Thus putting $h=4, k=1$ and $\beta_{k, p}=0$ (if $p \mid f$ ) in Theorem 5.3 we obtain (i).

If $d=-220$, then $f=2, d_{0}=d / f^{2}=-55,\left(\frac{d_{0}}{2}\right)=1$ and $h\left(d / 2^{2}\right)=$ $h\left(d_{0}\right)=h(-55)=4$. Note that

$$
\begin{aligned}
H(-220) & =\{[1,0,55],[7,2,8],[5,0,11],[7,-2,8]\} \\
H(-55) & =\{[1,1,14],[2,1,7],[4,3,4],[2,-1,7]\},
\end{aligned}
$$

and for any prime $p, p \in R(A) \Leftrightarrow p \in R([7,2,8]) \Leftrightarrow\left(\frac{p}{11}\right)=\left(\frac{p}{5}\right)=-1$. Now putting $d=-220, h=4, k=1, a_{2}=1$ and $a_{5}=a_{11}=2$ in Theorem 5.3 and then applying the above we see that (ii) holds.

If $d=-252$, then $f=6, d_{0}=d / f^{2}=-7, I=[1,0,63], A=[8,6,9]$, $A^{2}=[7,0,9]$, and for any prime $p, p \in R(A) \Leftrightarrow p>2$ and $p \equiv 2,8,11$ $(\bmod 21)$. Let $p$ be a prime dividing $f$, then $p=2$ or 3 . Note that $H(-63)=$ $\{[1,1,16],[2,1,8],[4,1,4],[2,-1,8]\},\left(\frac{d_{0}}{2}\right)=\left(\frac{-7}{2}\right)=1$ and $h\left(d / 3^{2}\right)=$ $h(-28)=2$. Putting $d=-252, h=4, k=1, a_{2}=1, a_{7}=2$ and $\beta_{1,3}=0$ in Theorem 5.3 and then applying the above we obtain the result.

By the above, the theorem is proved.

TheOREM 8.2. Let $s \in \mathbb{C}$ with $\operatorname{Re}(s)>1$. Then

$$
\text { (i) } \begin{aligned}
\sum_{n=1}^{\infty} \frac{\phi_{3}(n)}{n^{s}}= & \frac{1}{1+7^{-s}} \prod_{\substack{p \equiv 3,5,6(\bmod 7) \\
p \neq 3}} \frac{1}{1-p^{-2 s}} \prod_{p \equiv 2,8,11(\bmod 21)} \frac{1}{1+p^{-2 s}} \\
& \times \prod_{p=x^{2}+x y+16 y^{2}} \frac{1}{\left(1-p^{-s}\right)^{2}} \prod_{p=4 x^{2}+x y+4 y^{2} \neq 7} \frac{1}{\left(1+p^{-s}\right)^{2}},
\end{aligned}
$$


(ii) $\sum_{n=1}^{\infty} \frac{\phi_{4}(n)}{n^{s}}=\frac{1}{1+5^{-s}} \prod_{p \equiv 11,13,17,19(\bmod 20)} \frac{1}{1-p^{-2 s}}$

$$
\times \prod_{p \equiv 3,7(\bmod 20)} \frac{1}{1+p^{-2 s}} \prod_{p=x^{2}+20 y^{2}} \frac{1}{\left(1-p^{-s}\right)^{2}}
$$

$$
\times \prod_{p=4 x^{2}+5 y^{2} \neq 5} \frac{1}{\left(1+p^{-s}\right)^{2}}
$$

(iii) $\sum_{n=1}^{\infty} \frac{\phi_{8}(n)}{n^{s}}=\prod_{p \equiv 5,7(\bmod 8)} \frac{1}{1-p^{-2 s}} \prod_{p \equiv 3(\bmod 8)} \frac{1}{1+p^{-2 s}}$

$$
\times \prod_{p=x^{2}+32 y^{2}} \frac{1}{\left(1-p^{-s}\right)^{2}} \prod_{p=4 x^{2}+4 x y+9 y^{2}} \frac{1}{\left(1+p^{-s}\right)^{2}},
$$

(iv) $\sum_{n=1}^{\infty} \frac{\phi_{12}(n)}{n^{s}}=\prod_{p \equiv 3(\bmod 4)} \frac{1}{1-p^{-2 s}} \prod_{p \equiv 5(\bmod 12)} \frac{1}{1+p^{-2 s}}$

$$
\times \prod_{p=x^{2}+36 y^{2}} \frac{1}{\left(1-p^{-s}\right)^{2}} \prod_{p=4 x^{2}+9 y^{2}} \frac{1}{\left(1+p^{-s}\right)^{2}} .
$$

Proof. By Theorem 8.1(i) and the proof of Theorem 4.5 we obtain the result.

REMARK 8.1. In his lost notebook (see [R2]), Ramanujan conjectured the Euler product for $\sum_{n=1}^{\infty} \phi_{3}(n) n^{-s}$. But his formula is erroneous. So Rangachari's proof of this result is also somewhat wrong. In [R1] Ramanujan conjectured Theorem 8.2(iv). We have established these results in a unified and natural way.

Theorem 8.3. Let $s \in \mathbb{C}$ with $\operatorname{Re}(s)>1$. Then

$$
\text { (i) } \begin{aligned}
\sum_{n=1}^{\infty} & \frac{f_{1}(1,5 ; n)+f_{1}(3,5 ; n)}{n^{s}} \\
= & \frac{1}{1+3^{-s}} \cdot \frac{1}{1+13^{-s}} \prod_{\left(\frac{p}{13}\right)=-\left(\frac{p}{3}\right)} \frac{1}{1-p^{-2 s}} \prod_{\left(\frac{p}{13}\right)=\left(\frac{p}{3}\right)=-1} \frac{1}{1+p^{-2 s}} \\
& \times \prod_{p=x^{2}+x y+10 y^{2}} \frac{1}{\left(1-p^{-s}\right)^{2}} \prod_{p=3 x^{2}+3 x y+4 y^{2} \neq 3,13} \frac{1}{\left(1+p^{-s}\right)^{2}},
\end{aligned}
$$


(ii) $\sum_{n=1}^{\infty} \frac{f_{8}(1,5 ; n)+f_{8}(3,5 ; n)}{n^{s}}$

$$
=\prod_{p \equiv 3(\bmod 4)} \frac{1}{1-p^{-2 s}} \prod_{p \equiv 5(\bmod 8)} \frac{1}{1+p^{-2 s}} \prod_{p=x^{2}+64 y^{2}} \frac{1}{\left(1-p^{-s}\right)^{2}}
$$$$
\times \prod_{p=4 x^{2}+4 x y+17 y^{2}} \frac{1}{\left(1+p^{-s}\right)^{2}},
$$

(iii) $\sum_{n=1}^{\infty} \frac{f_{20}(1,5 ; n)+f_{20}(3,5 ; n)}{n^{s}}$

$=\prod_{p \equiv 3(\bmod 4)} \frac{1}{1-p^{-2 s}} \prod_{p \equiv 13,17(\bmod 20)} \frac{1}{1+p^{-2 s}} \prod_{p=x^{2}+100 y^{2}} \frac{1}{\left(1-p^{-s}\right)^{2}}$

$$
\times \prod_{p=4 x^{2}+25 y^{2}} \frac{1}{\left(1+p^{-s}\right)^{2}} .
$$

Proof. From [SW, Proposition 11.1(i)] we know that $H(d)$ is a cyclic group of order 4 for $d \in\{-39,-256,-400\}$. Actually,

$$
\begin{aligned}
H(-39) & =\{[1,1,10],[2,1,5],[3,3,4],[2,-1,5]\}, \\
H(-256) & =\{[1,0,64],[5,2,13],[4,4,17],[5,-2,13]\}, \\
H(-400) & =\{[1,0,100],[8,4,13],[4,0,25],[8,-4,13]\} .
\end{aligned}
$$

Thus, by Theorem 2.4 we have

$$
\begin{aligned}
f_{1}(1,5 ; n)+f_{1}(3,5 ; n) & =(R(1,1,10 ; n)-R(4,3,3 ; n)) / 2=F([2,1,5], n), \\
f_{8}(1,5 ; n)+f_{8}(3,5 ; n) & =(R(1,0,64 ; n)-R(4,-4,17 ; n)) / 2 \\
& =F([5,2,13], n)
\end{aligned}
$$

and

$$
\begin{aligned}
f_{20}(1,5 ; n)+f_{20}(3,5 ; n) & =(R(1,0,100 ; n)-R(4,-16,41 ; n)) / 2 \\
& =F([8,4,13], n) .
\end{aligned}
$$

For a prime $p$ it is clear that

$$
\begin{aligned}
& p \in R([2,1,5]) \Leftrightarrow\left(\frac{p}{13}\right)=\left(\frac{p}{3}\right)=-1, \\
& p \in R([5,2,13]) \Leftrightarrow p \equiv 5(\bmod 8), \\
& p \in R([8,4,13]) \Leftrightarrow p \equiv 13,17(\bmod 20) .
\end{aligned}
$$

Now combining all the above with Theorem 8.1(i) in the cases $d=$ $-39,-256,-400$ yields the result. 
REMARK 8.2. By [SW, Theorems 10.2 and 11.1] and the proofs of Theorems 7.3 and 8.3 , we may obtain explicit formulas for $f_{k}(1,5 ; n)+f_{k}(3,5 ; n)$ in the cases $k=1,2,8,20$.

THEOREM 8.4. Let $d<0$ be a discriminant such that $H(d)=\left\{I, A, A^{2}, A^{3}\right\}$ with $A^{4}=I$. (The values of such $d$ are given in [SW, Proposition 11.1(i)].) Let $f$ be the conductor of $d$ and $s \in \mathbb{C}$ with $\operatorname{Re}(s)>1$. Then

$$
\begin{aligned}
L\left(A^{2}, s\right)= & c(d, s) \prod_{p \mid d, p \nmid f} \frac{1}{1-p^{-s}} \prod_{\left(\frac{d}{p}\right)=-1} \frac{1}{1-p^{-2 s}} \\
& \times \prod_{\substack{p \in R(I) \cup R\left(A^{2}\right) \\
p \nmid d}} \frac{1}{\left(1-p^{-s}\right)^{2}} \prod_{p \in R(A)} \frac{1}{\left(1+p^{-s}\right)^{2}},
\end{aligned}
$$

where

$$
c(d, s)= \begin{cases}1+2^{1-2 s} & \text { if } d=-128,-256 \\ \frac{1+2^{1-s}+2^{1-2 s}}{\left(1+2^{-s}\right)^{2}} & \text { if } d=-220,-252 \\ \frac{1+2^{-s}+2^{1-2 s}}{1+2^{-s}} & \text { if } d=-80,-144,-208,-400,-592, \\ 1 & \text { otherwise. }\end{cases}
$$

Proof. By Theorem 5.3, the result is true when $f=1$. Now suppose $f>1$ and $d_{0}=d / f^{2}$. Let $p$ be a prime such that $p \mid f$. Suppose $p^{\alpha_{p}} \| f, h_{p}=$ $h\left(d / p^{2 \alpha_{p}}\right)$ and $\beta_{2, p}$ is the maximum $j \in\left\{0,1, \ldots, \alpha_{p}\right\}$ such that $2 \mid h\left(d / p^{2 j}\right)$. From [SW, Proposition 11.1(i)] we know that all possible $(d, f)$ with $f>1$ are given below:

$$
\begin{aligned}
(d, f)= & (-63,3),(-80,2),\left(-128,2^{2}\right),(-144,2 \cdot 3),(-171,3), \\
& (-196,7),(-208,2),(-220,2),(-252,2 \cdot 3),\left(-256,2^{3}\right), \\
& (-275,5),(-363,11),(-387,3),(-400,2 \cdot 5),(-475,5), \\
& (-507,13),(-592,2),(-603,3),(-1467,3) .
\end{aligned}
$$

From [SW, Proposition 11.1, Lemma 9.2 and (9.3)] we also have

$$
h_{p}=h\left(\frac{d}{p^{2 \alpha}}\right)=\left\{\begin{aligned}
1 \quad & \text { if } d \notin\{-80,-144,-208,-220,-252,-400,-592\} \\
& \quad \text { or } p>2, \\
2 & \text { if } d \in\{-80,-144,-208,-400,-592\} \text { and } p=2, \\
4 & \text { if } d \in\{-220,-252\} \text { and } p=2 .
\end{aligned}\right.
$$

If $p>2$, then $h_{p}=h\left(d / p^{2}\right)=1$. Thus $4 \nmid 2 h_{p}, \beta_{2, p}=0$ and hence $\left(1-p^{(1-2 s)\left(1+\beta_{2, p}\right)}\right) /\left(1-p^{1-2 s}\right)=1$. Thus applying Theorem 5.3 we see that the result is true in the cases $d=-63,-171,-196,-275,-363,-387$, $-475,-507,-603,-1467$. 
If $d=-128,-256$, then $p=2, p \mid d_{0}, h_{p}=1,4 \nmid 2 h_{p}$ and $\beta_{2, p}=1$, thus putting $d=-128,-256$ and $k=2$ in Theorem 5.3 we obtain the result.

If $d=-220$, then $p=f=2, h_{p}=h(-55)=4,4 \mid 2 h_{p},\left(\frac{d_{0}}{p}\right)=\left(\frac{-55}{2}\right)=1$, thus putting $d=-220, k=2$ and $a_{2}=1$ in Theorem 5.3 yields the result.

If $d=-252$, then $f=6, d_{0}=-63$ and so $p=2,3$. Observe that $h_{2}=h(-63)=4,4 \mid 2 h_{2}$ and $\left(\frac{d_{0}}{2}\right)=\left(\frac{-63}{2}\right)=1$. Putting $d=-252$ and $k=2$ in Theorem 5.3 gives the result.

If $d=-80,-144,-208,-400,-592$, then $2 \mid f$. Let $p=2$. Then $\alpha_{p}=1$, $h_{p}=2,4\left|2 h_{p}, p\right| d_{0}$ and $p$ is represented by the generator of $H\left(d / p^{2}\right)$ by [SW, Table 9.1]. Thus applying Theorem 5.3 and the above we obtain the result.

Combining the above we prove the theorem.

We remark that the conditions for $p \in R(I) \cup R\left(A^{2}\right)$ or $p \in R(A)$ in Theorem 8.4 can be described by certain congruence conditions.

\section{References}

[A] C. Adiga, On the representations of an integer as a sum of two or four triangular numbers, Nihonkai Math. J. 3 (1992), 125-131.

[B] B. C. Berndt, Ramanujan's Notebooks (Part III), Springer, New York, Berlin, 1991.

[BO] B. C. Berndt and K. Ono, Ramanujan's unpublished manuscript on the partition and tau functions with proofs and commentary, Sém. Lothar. Combin. 42 (1999), 63 pp.; also in: The Andrews Festschrift, D. Foata and G.-N. Han (eds.), Springer, Berlin, 2001, pp. 39-110.

[D] L. E. Dickson, History of the Theory of Numbers (Vol. III), Chelsea, New York, 1952, p. 42.

[Di] P. G. L. Dirichlet, Lectures on Number Theory (Supplements by R. Dedekind), transl. by J. Stillwell, Amer. Math. Soc., 1999, 217-221.

[HW] G. H. Hardy and E. M. Wright, An Introduction to the Theory of Numbers, 5th ed., Oxford Univ. Press, Oxford, 1981, pp. 242, 282-286, 314.

[H] L. K. Hua, Introduction to Number Theory, Springer, Berlin, 1982, p. 282.

[HKW] J. G. Huard, P. Kaplan and K. S. Williams, The Chowla-Selberg formula for genera, Acta Arith. 73 (1995), 271-301.

[IR] K. Ireland and M. Rosen, A Classical Introduction to Modern Number Theory, Springer, New York, 1982, pp. 279-282.

[KW1] P. Kaplan and K. S. Williams, The genera representing a positive integer, Acta Arith. 102 (2002), 353-361.

[KW2] - - - On the number of representations of a positive integer by a binary quadratic form, ibid. 114 (2004), 87-98.

[L] A. M. Legendre, Traité des Fonctions Elliptiques, Vol. 3, Paris, 1832.

[MOS] W. Magnus, F. Oberhettinger and R. P. Soni, Formulas and Theorems for the Special Functions of Mathematical Physics, 3rd ed., Springer, New York, 1966, pp. 256-259.

[M] L. J. Mordell, On Mr Ramanujan's empirical expansions of modular functions, Proc. Cambridge Philos. Soc. 19 (1917), 117-124. 
[MW] H. Muzaffar and K. S. Williams, Evaluation of Weber's functions at quadratic irrationalities, JP J. Algebra Number Theory Appl. 4 (2004), 209-259.

[ORW] K. Ono, S. Robins and P. T. Wahl, On the representation of integers as sums of triangular numbers, Aequationes Math. 50 (1995), 73-94.

[R1] S. Ramanujan, On certain arithmetical functions, Trans. Cambridge Philos. Soc. 22 (1916), 159-184.

[R2] - The Lost Notebook and Other Unpublished Papers, Narosa, New Delhi, 1988.

[Ra] S. S. Rangachari, Ramanujan and Dirichlet series with Euler products, Proc. Indian Acad. Sci. Math. Sci. 91 (1982), 1-15.

[SC] A. Selberg and S. Chowla, On Epstein's zeta function, J. Reine Angew. Math. 227 (1967), 86-110.

[SW] Z. H. Sun and K. S. Williams, On the number of representations of $n$ by $a x^{2}+$ $b x y+c y^{2}$, Acta Arith. 122 (2006), 101-171.

[W] K. S. Williams, Some Lambert series expansions of products of theta functions, Ramanujan J. 3 (1999), 367-384.

[WH] K. S. Williams and R. H. Hudson, Representation of primes by the principal form of discriminant $-D$ when the class number $h(-D)$ is 3 , Acta Arith. 57 (1991), 131-153.

[ZW] N. Y. Zhang and K. S. Williams, On the Epstein zeta function, Tamkang J. Math. 26 (1995), 165-176.

Department of Mathematics

Huaiyin Teachers College

Huaian, Jiangsu 223001, P.R. China

E-mail: hyzhsun@public.hy.js.cn

http://www.hytc.cn/xsjl/szh
Centre for Research in Algebra and Number Theory School of Mathematics and Statistics Carleton University Ottawa, Ontario K1S 5B6, Canada

E-mail: williams@math.carleton.ca http://mathstat.carleton.ca/ ${ }^{\sim}$ williams

Received on 24.2.2005

and in revised form on 10.6.2005 